\title{
EIGENFRACTURE: AN EIGENDEFORMATION APPROACH TO VARIATIONAL FRACTURE*
}

\author{
BERND SCHMIDT ${ }^{\dagger},{\text { FERNANDO FRATERNALI }{ }^{\ddagger}, \text { AND MICHAEL ORTIZ }}^{\S}$
}

\begin{abstract}
We propose an approximation scheme for a variational theory of brittle fracture. In this scheme, the energy functional is approximated by a family of functionals depending on a small parameter and on two fields: the displacement field and an eigendeformation field that describes the fractures that occur in the body. Specifically, the eigendeformations allow the displacement field to develop jumps that cost no local elastic energy. However, this local relaxation requires the expenditure of a certain amount of fracture energy. We provide a construction, based on the consideration of $\varepsilon$-neighborhoods of the support of the eigendeformation field, for calculating the right amount of fracture energy associated with the eigendeformation field. We prove the $\Gamma$-convergence of the eigendeformation functional sequence, and of finite element approximations of the eigendeformation functionals, to the Griffith-type energy functional introduced in Francfort and Marigo [J. Mech. Phys. Solids, 46 (1998), pp. 1319-1342]. This type of convergence ensures the convergence of eigendeformation solutions, and of finite element approximations thereof, to brittle-fracture solutions. Numerical examples concerned with quasi-static mixed-mode crack propagation illustrate the versatility and robustness of the approach and its ability to predict crack-growth patterns in brittle solids.
\end{abstract}

Key words. brittle fracture, eigendeformation, two-field approximation, $\Gamma$-convergence, mesh anisotropy correction, variational element erosion

AMS subject classifications. 74R10, 49J45, 65K10, 74G15

DOI. $10.1137 / 080712568$

1. Introduction. Several free discontinuity models generalizing the classical Griffith theory of brittle fracture have appeared in the literature over the past few years (generalized Griffith theories; see, e.g., Francfort and Marigo [30]; Dal Maso and Toader [26]; Chambolle [16]; Francfort and Larsen [31]; Dal Maso, Francfort, and Toader [27]). The term free discontinuity problems was first introduced by De Giorgi and Ambrosio [28] to denote problems where the solution can have discontinuity points and, in addition, the jump sets of the solutions are a priori unknown (see also Ambrosio, Fusco, and Pallara [5]; Dal Maso [24]; Braides [12], [11]). As well as from fracture mechanics, examples are derived from image processing, shape optimization, and liquid crystals. In fracture mechanics, free discontinuities describe the crack pattern of the body.

Generalized Griffith models deal with minimization of energy functionals composed of bulk and surface terms, the latter being defined over a set, the jump set, of codimension 1. This jump set forms an argument for the functional to be minimized together with the displacement field of the body. Mathematical research in the field has found sufficient conditions in terms of functional setting (in spaces of special functions with bounded variation: SBV, SBD, GSBV), topology of the crack front,

\footnotetext{
* Received by the editors January 5, 2008; accepted for publication (in revised form) October 21, 2008; published electronically February 6, 2009.

http://www.siam.org/journals/mms/7-3/71256.html

${ }^{\dagger}$ Zentrum Mathematik, Technische Universität München, Boltzmannstr. 3, 85748 Garching, Germany (schmidt@ma.tum.de).

${ }^{\ddagger}$ Department of Civil Engineering, University of Salerno, 84084 Fisciano (SA), Italy (fraterna@ caltech.edu).

$\S$ Division of Engineering and Applied Science, California Institute of Technology, Pasadena, CA 91125 (ortiz@aero.caltech.edu).
} 
shape, and growth properties of bulk and surface energies, which ensure existence of solutions. The original variational model by Francfort and Marigo [30] considers a global minimization problem and admits only displacement-type loads (hard-device conditions). Inclusion of body forces and surface tractions has been carried out by Dal Maso, Francfort, and Toader [27] under special assumptions.

The Francfort-Marigo variational model was originally developed for the study of global energy minimization problems. Therefore, its application to fracture evolution requires additional development in order to properly account for the no-healing irreversibility constraint in models for crack evolution. The most common approach so far to the analysis of rate-independent fracture processes consists of the minimization of incremental energy functionals that geometrically or energetically constrain crack increments in order to enforce irreversibility and then taking the limit as the time step goes to zero [26], [16], [31], [27], [37]. An alternative approach is to regard fracture as a dissipative process with dissipation concentrated at the crack front [38]. In this approach, the crack front velocity is determined by a kinetic law in terms of the local driving force, and the entire trajectories of the system, including the crack paths, follow as minimizers of energy-dissipation functionals [40].

Several numerical approximations of generalized Griffith models have also been formulated, including both continuous (weak) and discontinuous (strong) approaches, with discontinuities replaced by sharp gradients in the first case. It has been shown that convergence of discrete solutions necessarily requires mesh adaption and/or nonlocal approaches, in order to avoid unrecoverable mesh-dependent anisotropy effects (cf. Bourdin, Francfort, and Marigo [10]; Bourdin and Chambolle [9]; Cortesani and Toader [21]; Chambolle and Dal Maso [19]; Lussardi and Negri [39]). Mesh adaption in this context may regard relocation of nodes ( $r$-adaption), size-refinement ( $h$-adaption), and/or topology optimization. For two-dimensional problems, finite element models with adaptive embedded discontinuities have been proposed by Negri [44] and Giacomini and Ponsiglione [36], while r-adaptive approaches with interelement discontinuities have been formulated by Angelillo, Babilio, and Fortunato [6] and Fraternali [32]. Weak approximations employing Ambrosio-Tortorelli regularizations [3] (Bourdin, Francfort, and Marigo [10]) or smeared cracks (Negri [43], [45], [46]) have also appeared.

This paper is concerned with an approximation scheme to generalized Griffith models of variational fracture based on the notion of eigendeformation. Eigendeformations (cf., e.g., [42]) are widely used in mechanics to describe deformation modes that cost no local energy. In the present context, the resulting approximating functionals

$$
E_{\varepsilon}(u, \gamma)=\int_{\Omega} W(\mathcal{E} u-\gamma)+\frac{G}{2 \varepsilon}\left|\{\gamma \neq 0\}_{\varepsilon}\right|
$$

depend on two fields: the displacement field $u$ and an eigendeformation field $\gamma$ that describes the fractures that occur in the body. Specifically, eigendeformations allow the displacement field to develop jumps at no cost in local elastic energy. However, this local relaxation comes at the expense of a certain amount of fracture energy. The crux of the present approach is a construction that delivers the right amount of fracture energy attendant to an eigendeformation field. To this end, we introduce a small parameter $\varepsilon$ and compute the fracture energy from the volume of the $\varepsilon$-neighborhood of the support of the eigendeformation field, suitably scaled by $1 / \varepsilon$. We note that other two-field approximation schemes for brittle fracture, most notably the Ambrosio-Tortorelli scheme [3], [11], have been proposed in the past, but the use 
of eigendeformations to describe brittle fracture in a variational framework does not appear to have been pursued heretofore. As a matter of convenience, the eigendeformation field can variously be chosen to be diffuse, representing a damaged volume of material, or concentrated on rectifiable surfaces, representing sharp cracks. We also note that damage regularizations of brittle fracture [13], [12], [11], [45] have been proposed in the past and shown to be convergent.

We begin by formulating and proving our main $\Gamma$-convergence result in one dimension (cf. section 2). This serves as an instructive and introductory special case, which already shows the main features of the proposed approximation scheme. It is also a first step of the proof of the general $d$-dimensional result: an important technical tool are certain slicing techniques in spaces of bounded variation $(S B V)$, and bounded deformation $(S B D)$, which have proved very useful for free discontinuity problems in higher dimensions (see, e.g., the pioneering work [2], [3] or [12, Chapter 15$]$ ). In section 3 we briefly review the definition and the slicing, compactness, and density properties of the spaces $S B V$ and $S B D$, which model our $d$-dimensional displacement fields. The general $\Gamma$-convergence and compactness result is then stated and proved in section 4 . The topology on the space of our two-field variables $(u, \gamma)$, consisting of the displacement field $u$ and the eigendeformation field $\gamma$, is derived from $L^{1}$-convergence in $u$ and the convergence in the flat norm, resp., with respect to suitable negative Sobolev norms, in the second variable $\gamma$. With the help of strong density results, the $\Gamma$-lim sup-inequality is straightforward. Therefore, the main part of the proof consists of showing compactness for bounded energy sequences and the $\Gamma$-liminf-inequality. We also briefly discuss a corresponding strongly convergence scheme that can be proved along similar lines.

Owing to the variational character of the proposed model of fracture, finite element approximations follow readily by restriction of the energy functional to dense sequences of finite-dimensional interpolation spaces. The $\Gamma$-convergence of the discrete energy functionals is then straightforward, and we state the relevant results in section 5. Evidently, the choice of interpolation space is not unique, and different choices result in different finite element implementations of the general scheme. For purposes of illustration, we describe a particular implementation based on a special piecewise constant interpolation of the eigendeformations within elements. The adopted numerical scheme employs a direct search method and an energy descent element erosion technique for the computation of the crack path. It can be easily integrated with standard finite element codes, requiring repeated solution of elastic problems with trial eigendeformations and parameter tuning via preliminary numerical tests. A fixed mesh is employed and mesh-dependent anisotropic effects [43] are circumvented thanks to the construction of the $\varepsilon$-neighborhood of the crack set. The numerical examples in section 6 illustrate the versatility of the approach in problems involving mixed mode I-II and I-III crack growth. Other possible implementations are also briefly mentioned in the concluding remarks, section 7 .

2. Results in one dimension. Suppose a beam occupying the region $(0,1)$ in $\mathbb{R}$ is subjected to the displacement $u:(0,1) \rightarrow \mathbb{R}$. Allowing for brittle fracture, the Griffith-type energy associated with $u$ is

$$
E(u):=\int_{0}^{1}\left|u^{\prime}\right|^{2}+G \# J_{u},
$$

where $J_{u}$ denotes the set of fracture points in $(0,1)$. The natural functional space to consider these problems in is the space $P-W^{1,2}(0,1)$ of piecewise $W^{1,2}$-functions on 
$(0,1)$. It is defined as $P-W^{1,2}(0,1):=W^{1,2}(0,1)+P C(0,1)$, where $P C(0,1)$ denotes the space of piecewise constant functions. Note that $W^{1,2}(0,1)$ embeds into $C([0,1])$, so we may assume $u$ to be piecewise continuous with existing left- and right-hand limits $u(x \pm)$ for every $x \in(0,1)$.

The distributional derivative $D u$ of a function $u \in P-W^{1,2}(0,1)$ can be decomposed into a part $u^{\prime}$ which is absolutely continuous with respect to Lebesgue measure and a singular part $D^{s} u$ which is a finite sum of Dirac-measures. We study approximations to $u$ and $E(u)$ by functions that do not have a singular part. One way to do this is by mollifying $D^{s} u$ with some smooth convolution kernel $\rho_{\varepsilon}$, where $\operatorname{supp}\left(\rho_{\varepsilon}\right)=[-\varepsilon, \varepsilon]$ and $\varepsilon \rightarrow 0$. Observing that, for a Dirac-mass $\mu=a \delta_{x}$,

$$
\frac{1}{2 \varepsilon}|\{\rho * \mu \neq 0\}|=\frac{1}{2 \varepsilon}|(x-\varepsilon, x+\varepsilon)|=1,
$$

we are led to the following two-field functional:

$$
E_{\varepsilon}(u, \gamma):=\int_{0}^{1}\left|u^{\prime}-\gamma\right|^{2}+\frac{G}{2 \varepsilon}\left|\{\gamma \neq 0\}_{\varepsilon}\right| .
$$

Here $u^{\prime}$ is the strain of the beam and $\gamma$ can be seen as an approximation to $u^{\prime}$ in regions of very high strain, so that $u^{\prime}-\gamma$ is in the elastic range of the material. By $\{\gamma \neq 0\}_{\varepsilon}$ we denote the $\varepsilon$-neighborhood of $\{\gamma \neq 0\}$.

We expect $\min _{\gamma} E_{\varepsilon}$ to converge to the Griffith-type energy $E(u)=\int_{0}^{1}\left|u^{\prime}\right|^{2}+$ $G \# J_{u}$. To make this connection precise we choose a constant $K$ and define $E_{\varepsilon}$ : $L^{1}(0,1) \times \mathcal{M}(0,1) \rightarrow \mathbb{R} \cup\{\infty\}$ by

$$
E_{\varepsilon}(u, \gamma):=\left\{\begin{array}{lc}
\int_{0}^{1}\left|u^{\prime}-\gamma\right|^{2}+\frac{G}{2 \varepsilon}\left|\{\gamma \neq 0\}_{\varepsilon}\right| & \text { if } u \in W^{1,1}, \gamma \in L^{1} \text { such that } \\
\infty & \|u\|_{L^{\infty}} \leq K, \\
& \text { otherwise. }
\end{array}\right.
$$

Here $\mathcal{M}(0,1)$ denotes the set of finite Radon measures on $(0,1)$. The limiting functional is defined to be $E: L^{1}(0,1) \times \mathcal{M}(0,1) \rightarrow \mathbb{R} \cup\{\infty\}$,

$$
E(u, \gamma):=\left\{\begin{array}{lrl}
\int_{0}^{1}\left|u^{\prime}\right|^{2}+G \# J_{u} & \text { if } u \in P-W^{1,2},\|u\|_{L^{\infty}} \leq K \\
& \gamma=D^{s} u \\
\infty & \text { otherwise }
\end{array}\right.
$$

Remark. Note that the condition on $\|u\|_{L^{\infty}}$ is merely of a technical nature. In particular it prevents broken pieces of the body from escaping to infinity. The constant $K$, however, may be chosen arbitrarily large without affecting the following convergence and compactness results.

Before we state our main result in one dimension, we have to endow $L^{1} \times \mathcal{M}$ with a topology leading to the desired $\Gamma$-convergence result and simultaneously ensuring good compactness properties of low energy sequences. A natural choice for the first component is to choose the strong topology on $L^{1}$. A good choice for $\mathcal{M}$ turns out to be the topology induced by the flat norm, which-for general $d$-dimensional domains $\Omega$-is defined as follows: By $\mathcal{M}\left(\Omega, \mathbb{R}^{n}\right)$ we denote the set of $\mathbb{R}^{n}$-valued Radon measures on $\Omega$. The flat norm of a measure $\mu \in \mathcal{M}\left(\Omega, \mathbb{R}^{n}\right)$ is defined to be

$$
\|\mu\|_{\text {flat }}:=\sup \left\{\int_{\Omega} \varphi d \mu: \varphi: \Omega \rightarrow \mathbb{R}^{n} \operatorname{Lipschitz},|\varphi| \leq 1, \operatorname{Lip}(\varphi) \leq 1\right\} .
$$


Note that there is some freedom in the choice of the topology. In fact we prove that compactness even holds with respect to suitable negative Sobolev norms. As a consequence, also the $\Gamma$-convergence result holds true with respect to these stronger topologies. We also remark that-for general $d-\Gamma$-convergence is still true if $\mathcal{M}$ is endowed with the weak*-convergence of measures; compactness, however, fails as bounded energy sequences need not be bounded in $\mathcal{M}$ with respect to the total variation norm.

ThEOREM 2.1 ( $\Gamma$-convergence and compactness). Let $L^{1}(0,1)$ be equipped with the strong topology and $\mathcal{M}(0,1)$ with the topology induced by the flat norm $\|\cdot\|_{\text {flat }}$. Then $E_{\varepsilon} \Gamma$-converges to $E$ on $L^{1}(0,1) \times \mathcal{M}(0,1)$. Moreover, bounded energy sequences are compact in $W^{-1, q}(0,1)$ for all $q<\infty$ and in particular with respect to $\|\cdot\|_{\text {flat }}$. I.e., we have the following:

(i) Lower bound and compactness: If $\left(u_{\varepsilon}, \gamma_{\varepsilon}\right)$ is a sequence with $E_{\varepsilon}\left(u_{\varepsilon}, \gamma_{\varepsilon}\right) \leq C$, then, for a subsequence, there are $u \in P-W^{1,2}$ and $\gamma \in \mathcal{M}$ with $\gamma=D^{s} u$ such that $u_{\varepsilon} \rightarrow u$ in $L^{1}, \gamma_{\varepsilon} \rightarrow \gamma$ in the flat norm, and

$$
\liminf _{\varepsilon \rightarrow 0} E_{\varepsilon}\left(u_{\varepsilon}, \gamma_{\varepsilon}\right) \geq E(u, \gamma)
$$

In fact, $\gamma_{\varepsilon} \rightarrow \gamma$ in $W^{-1, q}$ for all $q<\infty$.

(ii) Recovery sequence: For all $(u, \gamma) \in L^{1} \times \mathcal{M}$ there exist $u_{\varepsilon} \in L^{1}$ and $\gamma_{\varepsilon} \in \mathcal{M}$ such that $u_{\varepsilon} \rightarrow u$ in $L^{1}, \gamma_{\varepsilon} \rightarrow \gamma$ in the flat norm, and

$$
\limsup _{\varepsilon \rightarrow 0} E_{\varepsilon}\left(u_{\varepsilon}, \gamma_{\varepsilon}\right) \leq E(u, \gamma)
$$

Proof of (ii). We may assume that $u \in P-W^{1,2}(0,1), J_{u}=\left\{x_{1}, \ldots, x_{N}\right\}$, $\|u\|_{L^{\infty}} \leq K$, and $\gamma=D^{s} u$. Let $\delta=\varepsilon^{2}$ and suppose that $\varepsilon$ is small enough that $2 \varepsilon+2 \delta<\min _{i}\left|x_{i+1}-x_{i}\right|$. Then define $\gamma_{\varepsilon} \in L^{1}$ by

$$
\gamma_{\varepsilon}(x)= \begin{cases}\frac{u\left(x_{i}+\delta\right)-u\left(x_{i}-\delta\right)}{2 \delta} & \text { for } x_{i}-\delta<x<x_{i}+\delta, \\ 0 & \text { otherwise }\end{cases}
$$

and $u_{\varepsilon} \in W^{1,1}$ by

$$
\begin{array}{ll}
u_{\varepsilon}^{\prime}(x)=\frac{u\left(x_{i}+\delta\right)-u\left(x_{i}-\delta\right)}{2 \delta} & \text { for } x_{i}-\delta<x<x_{i}+\delta, \\
u_{\varepsilon}(x)=u(x) & \text { for } x \notin \bigcup_{i}\left(x_{i}-\delta, x_{i}+\delta\right) .
\end{array}
$$

Clearly,

$$
\left\|u_{\varepsilon}-u\right\|_{L^{1}} \leq \int_{\bigcup_{i}\left(x_{i}-\delta, x_{i}+\delta\right)}\left|u_{\varepsilon}-u\right| \leq 2 K\left|\bigcup_{i}\left(x_{i}-\delta, x_{i}+\delta\right)\right| \rightarrow 0
$$

as $\varepsilon \rightarrow 0$. Moreover, it is straightforward to verify that

$$
\gamma_{\varepsilon} \rightarrow \sum_{i=1}^{N}\left(u\left(x_{i}+\right)-u\left(x_{i}-\right)\right) \delta_{x_{i}}=D^{s} u
$$

as $\varepsilon \rightarrow 0$ in the flat norm. Now noting that

$$
\begin{aligned}
E_{\varepsilon}\left(u_{\varepsilon}, \gamma_{\varepsilon}\right) & =\int_{0}^{1}\left|u_{\varepsilon}^{\prime}-\gamma_{\varepsilon}\right|^{2}+\frac{G}{2 \varepsilon}\left|\{\gamma \neq 0\}_{\varepsilon}\right| \\
& =\int_{(0,1) \backslash \bigcup_{i}\left(x_{i}-\delta, x_{i}+\delta\right)}\left|u^{\prime}\right|^{2}+\frac{G}{2 \varepsilon} N(2 \varepsilon+2 \delta) \rightarrow \int_{(0,1)}\left|u^{\prime}\right|^{2}+G N
\end{aligned}
$$

Copyright $@$ by SIAM. Unauthorized reproduction of this article is prohibited. 
as $\varepsilon \rightarrow 0$, we conclude the proof of (ii).

Proof of (i). Suppose $E_{\varepsilon}\left(u_{\varepsilon}, \gamma_{\varepsilon}\right) \leq C$, so in particular $\left|u_{\varepsilon}^{\prime}-\gamma_{\varepsilon}\right|$ is bounded in $L^{2}$. Since $u_{\varepsilon}$ is bounded in $L^{\infty}, \gamma_{\varepsilon}$ is bounded in $W^{-1, \infty}$ and thus converges, up to subsequences, weakly* to some $\gamma \in W^{-1, \infty}$. Write

$$
\left\{\gamma_{\varepsilon} \neq 0\right\}_{\varepsilon}=\left(r_{1}, s_{1}\right) \dot{\cup} \cdots \dot{\cup}\left(r_{n_{\varepsilon}}, s_{n_{\varepsilon}}\right)
$$

as a disjoint union of intervals $\left(s_{i}, r_{i}\right)$ with $\left|s_{i}-r_{i}\right| \geq \varepsilon$, where, by the energy bound, $n_{\varepsilon}$ is bounded independently of $\varepsilon$. So, if necessary passing to a subsequence, we may assume that $n=n_{\varepsilon}$ is independent of $\varepsilon$ and there exists a finite set $I=\left\{x_{1}, \ldots, x_{n}\right\} \subset$ $(0,1)$ such that $r_{i}, s_{i} \rightarrow x_{i}$. In particular, the distribution $\gamma$ is supported on $I$.

Let $U \subset \subset(0,1) \backslash I$ be an open set. For $\varepsilon$ sufficiently small $\left\{\gamma_{\varepsilon} \neq 0\right\}_{\varepsilon} \cap U=\emptyset$ and hence $\int_{U}\left|u_{\varepsilon}^{\prime}\right|^{2} \leq C$. Since $\left\|u_{\varepsilon}\right\|_{L^{\infty}} \leq K$, we obtain that, for a further subsequence, $u_{\varepsilon} \rightarrow u$ in $W^{1,2}(U)$ for some $u \in W^{1,2}(U)$ with $\|u\|_{L^{\infty}(U)} \leq K$. This being true for all such $U$, choosing a diagonal subsequence we find that in fact $u_{\varepsilon} \rightarrow u$ in $L^{q}(0,1)$ for all $q<\infty$ and $u \in P-W^{1,2}(0,1),\|u\|_{L^{\infty}(0,1)} \leq K$ with $J_{u} \subset I$.

It follows in particular that $u_{\varepsilon}^{\prime}=D u_{\varepsilon} \rightarrow D u=u^{\prime}+D^{s} u$ in $W^{-1, q}$. On the other hand, $u_{\varepsilon}^{\prime}-\gamma_{\varepsilon}$ converges weakly in $L^{2}$ for a suitable subsequence, so $\gamma_{\varepsilon} \rightarrow \gamma$ in $W^{-1, q}$ and $D u-\gamma \in L^{2}$. In particular, $\gamma$ lies in $\mathcal{M}(0,1)$ and, being supported on $I$, is a finite sum of Dirac-deltas. This implies that $\gamma=D^{s} u$.

Summarizing, we have seen that there exists $u \in P-W^{1,2}(0,1)$ with $\|u\|_{L^{\infty}(0,1)} \leq$ $K$ such that, up to subsequences, $u_{\varepsilon} \rightarrow u$ in $L^{1}(0,1)$ and $\gamma_{\varepsilon} \rightarrow \gamma=D^{s} u$ in $W^{-1, q}, q<$ $\infty$, and thus also in the flat norm. By passing, if necessary, to a suitable subsequence we may assume that $u_{\varepsilon} \rightarrow u$ a.e. It remains to prove the liminf-inequality. To this end it proves useful to introduce the localized energies $E_{\varepsilon}^{(r, s)}$ defined by

$$
E_{\varepsilon}^{(r, s)}\left(u_{\varepsilon}, \gamma_{\varepsilon}\right)=\int_{r}^{s}\left|u_{\varepsilon}^{\prime}-\gamma_{\varepsilon}\right|^{2}+\frac{G}{2 \varepsilon}\left|\left\{\gamma_{\varepsilon} \neq 0\right\}_{\varepsilon} \cap(r, s)\right|
$$

on subintervals $(r, s) \subset(0,1)$.

Let $J_{u}=\left\{y_{1}, \ldots, y_{N}\right\}, y_{1}<\cdots<y_{N}$, and choose $\delta>0$ so small that $\left|y_{i+1}-y_{i}\right| \geq$ $4 \delta$ and $\frac{\left(\left|u\left(y_{i}+\right)-u\left(y_{i}-\right)\right|-2 \delta\right)^{2}}{2 \delta} \geq G$ for all $i$. Then for each $y_{i}$ there are points $y_{i}^{\prime} \in$ $\left(y_{i}-\delta, y_{i}\right), y_{i}^{\prime \prime} \in\left(y_{i}, y_{i}+\delta\right)$ such that, for sufficiently small $\varepsilon$,

$$
\left|u_{\varepsilon}\left(y_{i}^{\prime}\right)-u\left(y_{i}-\right)\right|<\delta \quad \text { and } \quad\left|u_{\varepsilon}\left(y_{i}^{\prime \prime}\right)-u\left(y_{i}+\right)\right|<\delta .
$$

But then, for $\varepsilon$ sufficiently small, $E_{\varepsilon}^{\left(y_{i}^{\prime}-\varepsilon, y_{i}^{\prime \prime}+\varepsilon\right)}\left(u_{\varepsilon}, \gamma_{\varepsilon}\right) \geq G$. This is clear if $\gamma_{\varepsilon}$ does not vanish on $\left(y_{i}^{\prime}, y_{i}^{\prime \prime}\right)$. If $\gamma_{\varepsilon}=0$ a.e. on $\left(y_{i}^{\prime}, y_{i}^{\prime \prime}\right)$, it follows from

$$
\begin{aligned}
E_{\varepsilon}^{\left(y_{i}^{\prime}-\varepsilon, y_{i}^{\prime \prime}+\varepsilon\right)}\left(u_{\varepsilon}, \gamma_{\varepsilon}\right) & =\int_{y_{i}^{\prime}-\varepsilon}^{y_{i}^{\prime \prime}+\varepsilon}\left|u_{\varepsilon}^{\prime}\right|^{2} \geq \int_{y_{i}^{\prime}}^{y_{i}^{\prime \prime}}\left|u_{\varepsilon}^{\prime}\right|^{2} \geq \frac{1}{y_{i}^{\prime \prime}-y_{i}^{\prime}}\left(\int_{y_{i}^{\prime}}^{y_{i}^{\prime \prime}}\left|u_{\varepsilon}^{\prime}\right|\right)^{2} \\
& \geq \frac{\left(u_{\varepsilon}\left(y_{i}^{\prime \prime}\right)-u_{\varepsilon}\left(y_{i}^{\prime}\right)\right)^{2}}{y_{i}^{\prime \prime}-y_{i}^{\prime}} \geq \frac{\left(\left|u\left(y_{i}+\right)-u\left(y_{i}-\right)\right|-2 \delta\right)^{2}}{2 \delta} .
\end{aligned}
$$

Now suppose to the contrary that $u$ is absolutely continuous on $(r, s)$ with $|s-r|>\varepsilon$. Then

$$
E_{\varepsilon}^{(r, s)}\left(u_{\varepsilon}, \gamma_{\varepsilon}\right):=\int_{r}^{s}\left|u_{\varepsilon}^{\prime}-\gamma_{\varepsilon}\right|^{2}+\frac{G}{2 \varepsilon}\left|\{\gamma \neq 0\}_{\varepsilon} \cap(r, s)\right| \geq \min \left\{\int_{r}^{s}\left|u_{\varepsilon}^{\prime}\right|^{2}, \frac{G}{2}\right\}
$$

Copyright (c) by SIAM. Unauthorized reproduction of this article is prohibited. 
as can again be seen by estimating according to $\gamma_{\varepsilon}=0$ a.e. on $(r, s)$ or not. Let $n \in \mathbb{N}$ and set $r_{i}=r+\frac{i(s-r)}{n}$. For $n$ so large that $\int_{r_{i-1}}^{r_{i}}\left|u^{\prime}\right|^{2}<\frac{G}{2}$ for all $i=1, \ldots, n$, the same reasoning applied to $E_{\varepsilon}^{\left(r_{i-1}, r_{i}\right)}$ yields

$$
E_{\varepsilon}^{(r, s)}\left(u_{\varepsilon}, \gamma_{\varepsilon}\right) \geq \sum_{i=1}^{n} E_{\varepsilon}^{\left(r_{i-1}, r_{i}\right)}\left(u_{\varepsilon}, \gamma_{\varepsilon}\right) \geq \sum_{i=1}^{n} \min \left\{\int_{r_{i-1}}^{r_{i}}\left|u_{\varepsilon}^{\prime}\right|^{2}, \frac{G}{2}\right\}
$$

for $\varepsilon$ small enough. But then from $u_{\varepsilon} \rightarrow u$ in $L^{1}$ and $\int_{r_{i-1}}^{r_{i}}\left|u_{\varepsilon}^{\prime}\right|^{2} \leq C$ for all $i$ we can deduce that

$$
\begin{aligned}
\liminf _{\varepsilon \rightarrow 0} E_{\varepsilon}^{(r, s)}\left(u_{\varepsilon}, \gamma_{\varepsilon}\right) & \geq \sum_{i=1}^{n} \min \left\{\liminf _{\varepsilon \rightarrow 0} \int_{r_{i-1}}^{r_{i}}\left|u_{\varepsilon}^{\prime}\right|^{2}, \frac{G}{2}\right\} \\
& \geq \sum_{i=1}^{n} \min \left\{\int_{r_{i-1}}^{r_{i}}\left|u^{\prime}\right|^{2}, \frac{G}{2}\right\}=\int_{r}^{s}\left|u^{\prime}\right|^{2}
\end{aligned}
$$

Summarizing we find that indeed (with $y_{0}:=0, y_{N+1}:=1$ )

$$
\begin{aligned}
\liminf _{\varepsilon \rightarrow 0} E_{\varepsilon}\left(u_{\varepsilon}, \gamma_{\varepsilon}\right) & \geq \liminf _{\varepsilon \rightarrow 0} \sum_{i=1}^{N} E_{\varepsilon}^{\left(y_{i}^{\prime}-\varepsilon, y_{i}^{\prime \prime}+\varepsilon\right)}\left(u_{\varepsilon}, \gamma_{\varepsilon}\right)+\sum_{i=1}^{N+1} E_{\varepsilon}^{\left(y_{i-1}+\delta, y_{i}-\delta\right)}\left(u_{\varepsilon}, \gamma_{\varepsilon}\right) \\
& \geq G N+\int_{\bigcup_{i=1}^{N+1}\left(y_{i-1}+\delta, y_{i}-\delta\right)}\left|u^{\prime}\right|^{2} .
\end{aligned}
$$

Now letting $\delta \rightarrow 0$ we arrive at

$$
\liminf _{\varepsilon \rightarrow 0} E_{\varepsilon}\left(u_{\varepsilon}, \gamma_{\varepsilon}\right) \geq \int_{0}^{1}\left|u^{\prime}\right|^{2}+G \# J_{u} .
$$

3. Technical preliminaries: $\boldsymbol{S B V}$ and $\boldsymbol{S B D}$. In the general $d$-dimensional case, the domain of the elastic body is a Lipschitz domain $\Omega \subset \mathbb{R}^{d}$ and we consider deformations of bounded variation or deformation. Recall that $u \in L^{1}\left(\Omega, \mathbb{R}^{m}\right)$ is said to be an element of $B V\left(\Omega, \mathbb{R}^{m}\right)$ if its distributional derivative $D u$ is a finite $\mathbb{R}^{m \times d_{-}}$ valued Radon measure. Then $D u$ can be decomposed into an absolutely continuous part $\nabla u$ with respect to the Lebesgue measure $\mathcal{L}^{d}$ and a singular part $D^{s} u$. Assuming that the Cantor part of $D^{s} u$ vanishes we arrive at the space of special functions of bounded variation $S B V\left(\Omega, \mathbb{R}^{m}\right)$. If $u \in S B V\left(\Omega, \mathbb{R}^{m}\right)$, then $D u$ can be written as

$$
D u=\nabla u \mathcal{L}^{d}+\left(u^{+}-u^{-}\right) n_{u} \mathcal{H}^{d-1}\left\lfloor J_{u} .\right.
$$

Here $\mathcal{H}^{d-1}$ denotes the $(d-1)$-dimensional Hausdorff measure, $J_{u}$ is an $\mathcal{H}^{d-1}$-rectifiable subset of $\Omega$ (the "jump part" of $D u$ ), $n_{u}$ is the normal to $J_{u}$, and $u^{+}$, resp., $u^{-}$, are the one-sided limits of $u$ at $J_{u}$. We say that $u \in S B V^{p}\left(\Omega, \mathbb{R}^{m}\right)$ if in addition $\nabla u \in L^{p}$ and $\mathcal{H}^{d-1}\left(J_{u}\right)<\infty$. (See [5] for an exhaustive treatment of $B V$ and $S B V$ functions.)

Similarly we say that a function $u \in L^{1}\left(\Omega, \mathbb{R}^{d}\right)$ is of bounded deformation if the symmetrized distributional derivative $E u:=\frac{1}{2}\left((D u)^{T}+D u\right)$ is a finite $\mathbb{R}_{\mathrm{sym}}^{d \times d}$-valued Radon measure. If the Cantor part of $E u$ is zero, $u$ is said to be in $S B D(\Omega)$ and $E u$ can be decomposed as

$$
E u=\mathcal{E} u \mathcal{L}^{d}+E^{s} u=\mathcal{E} u \mathcal{L}^{d}+\left(u^{+}-u^{-}\right) \odot n_{u} \mathcal{H}^{d-1}\left\lfloor J_{u},\right.
$$

Copyright $\odot$ by SIAM. Unauthorized reproduction of this article is prohibited. 
where $\mathcal{E} u$ is the absolutely continuous part of $E u$ with respect to $\mathcal{L}^{d}, u^{ \pm}, n_{u}$, and $J_{u}$ are as before, and $a \odot b:=\frac{1}{2}(a \otimes b+b \otimes a)$; cf. [4]. $u$ is an element of $S B D^{p}(\Omega)$ if in addition $\mathcal{E} u \in L^{p}$ and $\mathcal{H}^{d-1}\left(J_{u}\right)<\infty$.

Next we briefly recall the basic slicing and compactness properties for the preceding function spaces that are required to prove the lim inf-inequality of our main $\Gamma$-convergence result. (For $S B V$ functions cf. [5]; for $S B D$ functions cf. [4], [7].) Let $\xi \in S^{d-1}$ and suppose $z \in \Omega$. Let

$$
\Omega^{z, \xi}=\{t \in \mathbb{R}: z+t \xi \in \Omega\} .
$$

For real valued functions $u: \Omega \rightarrow \mathbb{R}$, resp., vector valued functions $u: \Omega \rightarrow \mathbb{R}^{d}$, we define $u^{z, \xi}: \Omega^{z, \xi} \rightarrow \mathbb{R}$ by

$$
u^{z, \xi}(t)=u(z+t \xi), \quad \text { resp. }, \quad u^{z, \xi}(t)=u(z+t \xi) \cdot \xi .
$$

We then have the following slicing properties of $S B D$ (resp., $S B V$ ) functions.

TheOREm 3.1. Let $u \in S B D(\Omega)$. For a.e. $\xi \in S^{d-1}$ and $\mathcal{H}^{d-1}$-a.e. $z$ in the orthogonal complement $\Xi=\{x: x \cdot \xi=0\}$ the function $u^{z, \xi}$ belongs to $S B V\left(\Omega^{z, \xi}\right)$. Moreover,

$$
\begin{aligned}
& \xi^{T} \mathcal{E} u(z+t \xi) \xi=\left(u^{z, \xi}\right)^{\prime}(t) \text { for a.e. } t \in \Omega^{z, \xi}, \\
& J_{u^{z, \xi}}=\left(J_{u}\right)^{z, \xi}:=\left\{t \in \mathbb{R}: z+t \xi \in J_{u}\right\}, \\
& \int_{\Xi} \#\left(J_{u}\right)^{z, \xi} d \mathcal{H}^{d-1}(z)=\int_{J_{u}}\left|n_{u} \cdot \xi\right| d \mathcal{H}^{d-1} .
\end{aligned}
$$

An analogous result holds for $S B V$ functions: Just replace $\xi^{T} \mathcal{E} u(z+t \xi) \xi$ in the equation above by $\nabla u(z+t \xi) \cdot \xi$.

For the fundamental compactness result in $S B D$ (and $S B V$ ) suppose that $W$ is a positive definite quadratic form on the space $\mathbb{R}_{\mathrm{sym}}^{d \times d}$ of symmetric $d \times d$ matrices (resp., on $\mathbb{R}^{d}$ ).

THEOREM 3.2. Let $\left(u_{\varepsilon}\right)$ be a sequence in $S B D(\Omega)$ such that

$$
\int_{\Omega} W\left(\mathcal{E} u_{\varepsilon}\right) d x+\mathcal{H}^{d-1}\left(J_{u_{\varepsilon}}\right)+\left\|u_{\varepsilon}\right\|_{L^{\infty}} \leq C
$$

for some constant $C$ not depending on $\varepsilon$. Then there exist a subsequence (not relabeled) and a function $u \in S B D^{2}(\Omega)$ such that $u_{\varepsilon} \rightarrow u$ in $L^{1}(\Omega), \mathcal{E} u_{\varepsilon} \rightarrow \mathcal{E} u$ in $L^{2}\left(\Omega, \mathbb{R}^{d}\right)$, and

$$
\liminf _{\varepsilon \rightarrow 0} \int_{\Omega} W\left(\mathcal{E} u_{\varepsilon}\right) d x \geq \int_{\Omega} W\left(\mathcal{E} u_{\varepsilon}\right) d x \quad \text { and } \quad \liminf _{\varepsilon \rightarrow 0} \mathcal{H}^{d-1}\left(J_{u_{\varepsilon}}\right) \geq \mathcal{H}^{d-1}\left(J_{u}\right) .
$$

An analogous compactness theorem holds for $S B V$ functions.

The basic tool for the proof of the $\Gamma$-lim sup-inequalities are the following density results for $S B V$ and $S B D$ functions. Suppose $\mathcal{W}\left(\Omega, \mathbb{R}^{m}\right)$ is the space of all functions $u \in S B V\left(\Omega, \mathbb{R}^{m}\right)$ such that $J_{u}$ is a finite union of disjoint $(d-1)$-simplices and $u \in W^{k, \infty}\left(\Omega \backslash J_{u}, \mathbb{R}^{m}\right)$ for every $k$. The following theorem is found, e.g., in [20].

TheOrem 3.3. For every $u \in S B V^{2}\left(\Omega, \mathbb{R}^{m}\right) \cap L^{\infty}\left(\Omega, \mathbb{R}^{m}\right)$ there exist a sequence $u_{n} \in \mathcal{W}\left(\Omega, \mathbb{R}^{m}\right)$ such that $\left\|u_{n}\right\|_{L^{\infty}} \leq\|u\|_{L^{\infty}}$ and

$$
\begin{aligned}
& u_{n} \rightarrow u \text { strongly in } L^{1}\left(\Omega, \mathbb{R}^{m}\right), \\
& \nabla u_{n} \rightarrow \nabla u \text { strongly in } L^{2}\left(\Omega, \mathbb{R}^{m \times d}\right), \\
& \limsup _{n \rightarrow \infty} \mathcal{H}^{d-1}\left(J_{u_{n}}\right) \leq \mathcal{H}^{d-1}\left(J_{u}\right) .
\end{aligned}
$$

Copyright (c) by SIAM. Unauthorized reproduction of this article is prohibited. 
Combining this with the density results for $S B D$ functions first proved in [17] in two dimensions and extended in [18] to arbitrary dimensions, we obtain the following density result for $S B D$ functions. We cite it in the form used in [45], which is best suited for our purposes.

THEOREM 3.4. For every $u \in S B D^{2}(\Omega) \cap L^{\infty}\left(\Omega, \mathbb{R}^{d}\right)$ there exist a sequence $u_{n} \in \mathcal{W}\left(\Omega, \mathbb{R}^{d}\right)$ such that $\left\|u_{n}\right\|_{L^{\infty}} \leq\|u\|_{L^{\infty}}$ and

$$
\begin{aligned}
& u_{n} \rightarrow u \text { strongly in } L^{1}\left(\Omega, \mathbb{R}^{d}\right), \\
& \mathcal{E} u_{n} \rightarrow \mathcal{E} u \text { strongly in } L^{2}\left(\Omega, \mathbb{R}^{d \times d}\right), \\
& \limsup _{n \rightarrow \infty} \mathcal{H}^{d-1}\left(J_{u_{n}}\right) \leq \mathcal{H}^{d-1}\left(J_{u}\right) .
\end{aligned}
$$

A proof very similar to that of Proposition 2.5 in [35] shows that we may also impose suitable boundary conditions on the approximating sequence. Suppose that $\tilde{\Omega} \supset \Omega$ is a Lipschitz domain and the Dirichlet boundary of $\Omega$ is given by $\partial \Omega_{D}=$ $\tilde{\Omega} \cap \partial \Omega$. Let $g \in H^{r}(\tilde{\Omega}) \cap L^{\infty}, r \in \mathbb{N}$. Then we have the following variant of Theorem 3.4 accounting for the boundary data given by $g$.

TheOREM 3.5. For every $u \in S B D^{2}(\tilde{\Omega}) \cap L^{\infty}\left(\tilde{\Omega}, \mathbb{R}^{d}\right)$ with $u=g$ on $\tilde{\Omega} \backslash \Omega$ there exist a sequence of functions $u_{n} \in S B D^{2}(\Omega)$ and a sequence of neighborhoods $U_{n} \subset \tilde{\Omega}$ of $\tilde{\Omega} \backslash \Omega$ such that $u_{n}=g$ on $\tilde{\Omega} \backslash \Omega,\left.u_{n}\right|_{U_{n}} \in H^{r}\left(U_{n}\right),\left.u_{n}\right|_{\Omega_{n}} \in \mathcal{W}\left(\Omega_{n}, \mathbb{R}^{d}\right)$, where $\Omega_{n} \subset \Omega$ is some neighborhood of $\Omega \backslash U_{n}$, and such that $\left\|u_{n}\right\|_{L^{\infty}} \leq\|u\|_{L^{\infty}}$ and

$$
\begin{aligned}
& u_{n} \rightarrow u \text { strongly in } L^{1}\left(\Omega, \mathbb{R}^{d}\right), \\
& \mathcal{E} u_{n} \rightarrow \mathcal{E} u \text { strongly in } L^{2}\left(\Omega, \mathbb{R}^{d \times d}\right), \\
& \limsup _{n \rightarrow \infty} \mathcal{H}^{d-1}\left(J_{u_{n}}\right) \leq \mathcal{H}^{d-1}\left(J_{u}\right) .
\end{aligned}
$$

4. The general case. In this section we investigate deformations of bodies $\Omega \subset \mathbb{R}^{d}$ with Lipschitz boundaries $\partial \Omega$ in arbitrary dimensions. Then $u: \Omega \rightarrow \mathbb{R}^{d}$ and, correspondingly, $\gamma: \Omega \rightarrow \mathbb{R}^{d \times d}$. Note that analogous results hold for pure antiplane shear deformations that can be described by scalar valued functions $u$ and vector valued $\gamma$. In this setup it would be sufficient to work with $S B V$ instead of $S B D$ functions. Thanks to the largely parallel theory for $S B V$ and $S B D$ highlighted in the previous section, except for the obvious alternations like replacing $E u$ by $D u, \mathcal{E} u$ by $\nabla u$, etc., the following results and the corresponding proofs are literally the same.

4.1. Statement of the main result. In the general case the approximating deformations are described by functions $u \in W^{1,1}\left(\Omega, \mathbb{R}^{d}\right)$ and $\gamma \in L^{1}\left(\Omega, \mathbb{R}_{\mathrm{sym}}^{d \times d}\right)$, where $\mathbb{R}_{\text {sym }}^{d \times d}$ denotes the symmetric $d \times d$ matrices. Suppose $W$ is a positive definite quadratic form on $\mathbb{R}_{\mathrm{sym}}^{d \times d}$. In the applications to elasticity theory we usually have $d=1,2,3$.

For $u \in L^{1}\left(\Omega, \mathbb{R}^{d}\right)$ and $\gamma \in \mathcal{M}\left(\Omega, \mathbb{R}_{\mathrm{sym}}^{d \times d}\right)$ let

$$
E_{\varepsilon}(u, \gamma):=\left\{\begin{array}{lc}
\int_{\Omega} W(\mathcal{E} u-\gamma)+\frac{G}{2 \varepsilon}\left|\{\gamma \neq 0\}_{\varepsilon}\right| & \text { if } u \in W^{1,1},\|u\|_{L^{\infty}} \leq K, \\
\gamma \in L^{1}, & \text { otherwise, }
\end{array}\right.
$$

where $\{\gamma \neq 0\}$ is the complement of the zero-set of the precise representative of $\gamma$. The limiting functional is $E: L^{1}\left(\Omega, \mathbb{R}^{d}\right) \times \mathcal{M}\left(\Omega, \mathbb{R}_{\mathrm{sym}}^{d \times d}\right) \rightarrow \mathbb{R} \cup\{\infty\}$,

$$
E(u, \gamma):= \begin{cases}\int_{\Omega} W(\mathcal{E} u)+G \mathcal{H}^{2}\left(J_{u}\right) & \text { if } u \in S B D^{2},\|u\|_{L^{\infty}} \leq K, \\ & \gamma=E^{s} u, \\ \infty & \text { otherwise }\end{cases}
$$


Our main result is that $E_{\varepsilon} \Gamma$-converges to $E$. In the general case it is interesting to note that we can in addition impose the constraint that $\gamma=a \odot n$ be the symmetrization of a rank one function $x \mapsto a(x) \otimes n(x)$, where $a \in L^{1}\left(\Omega, \mathbb{R}^{d}\right)$ and $n$ is a unit vector field.

THEOREM 4.1 ( $\Gamma$-convergence and compactness). Let $L^{1}\left(\Omega, \mathbb{R}^{d}\right)$ be equipped with the strong and $\mathcal{M}\left(\Omega, \mathbb{R}_{\mathrm{sym}}^{d \times d}\right)$ with the flat topology. Then $E_{\varepsilon} \Gamma$-converges to $E$ on $L^{1}\left(\Omega, \mathbb{R}^{d}\right) \times \mathcal{M}\left(\Omega, \mathbb{R}_{\mathrm{sym}}^{d \times d}\right)$. Furthermore, bounded energy sequences are compact in this topology. I.e., we have the following:

(i) Lower bound and compactness: If $\left(u_{\varepsilon}, \gamma_{\varepsilon}\right)$ is a sequence with $E_{\varepsilon}\left(u_{\varepsilon}, \gamma_{\varepsilon}\right) \leq C$, then, for a subsequence, there are $u \in S B D^{2}$ and $\gamma \in \mathcal{M}$ with $\gamma=D^{s} u$ such that $u_{\varepsilon} \rightarrow u$ in $L^{1}, \gamma_{\varepsilon} \rightarrow \gamma$ in the flat norm, and

$$
\liminf _{\varepsilon \rightarrow 0} E_{\varepsilon}\left(u_{\varepsilon}, \gamma_{\varepsilon}\right) \geq E(u, \gamma)
$$

In fact, $\gamma_{\varepsilon} \rightarrow \gamma$ in $W^{-1, q}$ for all $q<2^{*}$, where $2^{*}=\frac{2 d}{d-2}$ if $d \geq 3$ and $2^{*}=\infty$ for $d=1,2$.

(ii) Recovery sequence: For all $(u, \gamma) \in L^{1} \times \mathcal{M}$ there exist $u_{\varepsilon} \in L^{1}$ and $\gamma_{\varepsilon} \in \mathcal{M}$ such that $u_{\varepsilon} \rightarrow u$ in $L^{1}, \gamma_{\varepsilon} \rightarrow \gamma$ in the flat norm, and

$$
\limsup _{\varepsilon \rightarrow 0} E_{\varepsilon}\left(u_{\varepsilon}, \gamma_{\varepsilon}\right) \leq E(u, \gamma) \text {. }
$$

Moreover, $\gamma_{\varepsilon}$ can be chosen as $\gamma_{\varepsilon}=a_{\varepsilon} \odot n_{\varepsilon}$ for suitable $a_{\varepsilon}, n_{\varepsilon} \in L^{1}\left(\Omega, \mathbb{R}^{d}\right)$ with $\left|n_{\varepsilon}\right|=1$.

Remarks.

(i) In particular, $E_{\varepsilon} \Gamma$-converges to $E$ with respect to the $L^{1} \times W^{-1, q}$-topology for all $q<2^{*}$.

(ii) In view of our numerical experiments in section 6 we also remark that, by Theorem 3.5, it is straightforward to include boundary values $g \in H^{1}\left(\tilde{\Omega}, \mathbb{R}^{d}\right)$, $\|g\|_{L^{\infty}} \leq K$ on a suitable Dirichlet boundary $\partial \Omega_{D}$ of $\Omega$ given by $\partial \Omega_{D}=\tilde{\Omega} \cap \partial \Omega$ for some Lipschitz domain $\tilde{\Omega} \supset \Omega$.

(iii) Thanks to our $L^{\infty}$-bound on the displacements it is also not hard to include body forces into our analysis. Surface tractions on the Neumann part $\partial \Omega_{N}$ of the boundary can be incorporated, too, once suitable assumptions are introduced to avoid cracking near this part of the boundary (e.g., by requiring that $\gamma_{\varepsilon}=0$ in a neighborhood of $\partial \Omega_{N}$; cf. [27]).

(iv) More generally, the above theorem can be easily extended to $W$ satisfying a standard $p$-growth assumption for $1<p<\infty$. (For $p>2$, one does not have that the simplicial components of $J_{u}$ in the definition of $u \in \mathcal{W}$ (cf. Theorems 3.3, 3.4, and 3.5) are disjoint. However, the construction of the recovery sequence in section 4.2 is not much harder in this case.)

Let us also remark that there is a corresponding strong approximation scheme that can be proved along the same lines as Theorem 4.1.

Theorem 4.2 ( $\Gamma$-convergence and compactness). Let

$$
\begin{aligned}
F(u) & := \begin{cases}\int_{\Omega} W(\mathcal{E} u)+G \mathcal{H}^{d-1}\left(J_{u}\right) & \text { if } u \in S B D^{2}(\Omega),\|u\|_{L^{\infty}} \leq K, \\
\infty & \text { otherwise, }\end{cases} \\
F_{\varepsilon}(u) & := \begin{cases}\int_{\Omega} W(\mathcal{E} u)+\frac{G}{2 \varepsilon}\left|\left(J_{u}\right)_{\varepsilon}\right| & \text { if } u \in S B D^{2}(\Omega),\|u\|_{L^{\infty}} \leq K, \\
\infty & \text { otherwise. }\end{cases}
\end{aligned}
$$

Then $F_{\varepsilon} \Gamma$-converges to $F$ with respect to the $L^{2}\left(\Omega, \mathbb{R}^{d}\right)$-metric. Furthermore, bounded energy sequences are compact in this topology. 
In fact, $F_{\varepsilon}$ being only a functional of the single variable $u$, the arguments in the following sections may even be considerably simplified to prove this result.

4.2. Recovery sequence. By a general density result in the theory of $\Gamma$-convergence and Theorem 3.4 it suffices to provide recovery sequences for $u \in \mathcal{W}\left(\Omega, \mathbb{R}^{d}\right)$. So suppose $u \in \mathcal{W}\left(\Omega, \mathbb{R}^{d}\right)$ and $J_{u}=J^{1} \cup \cdots \cup J^{m}$ for simplices $J^{1}, \ldots, J^{m}$ with normals $n_{1}, \ldots, n_{m}$. Let $\delta>0$ and by $J_{\delta}^{i}$ denote the $\delta$-neighborhood of $J^{i}$. Then define

$$
u_{\varepsilon}(x)=u(x) \quad \text { and } \quad a_{\varepsilon}(x)=0 \quad \forall x \in \Omega \backslash\left(J_{\delta}^{1} \cup \cdots \cup J_{\delta}^{m}\right) .
$$

On $J_{\delta}^{i}$ we define $u_{\varepsilon}$ - unambiguously for $\delta$ sufficiently small-by linear interpolation along $n_{i}$; i.e., if $x \in J_{\delta}^{i}$ lies on the segment $\left(x_{1}, x_{2}\right)$ for $x_{1}, x_{2} \in \partial J_{\delta}^{i}$ such that $x_{2}=x_{1}+\lambda n_{i}$ for some $\lambda \leq 2 \delta$, then

$$
u_{\varepsilon}(x)=\frac{\left|x-x_{1}\right|}{\left|x_{2}-x_{1}\right|} u\left(x_{2}\right)+\frac{\left|x_{2}-x\right|}{\left|x_{2}-x_{1}\right|} u\left(x_{1}\right) .
$$

For such $x$ we define

$$
n_{\varepsilon}(x)=n_{i} \quad \text { and } \quad a_{\varepsilon}(x)=\frac{u\left(x_{2}\right)-u\left(x_{1}\right)}{\left|x_{2}-x_{1}\right|} .
$$

If $x \notin J_{\delta}^{1} \cup \cdots \cup J_{\delta}^{m}$, we define $n_{\varepsilon}$ arbitrarily such that $\left|n_{\varepsilon}\right| \equiv 1$. Note that with this definition, due to our regularity assumptions on $u$,

$$
\left|\nabla u_{\varepsilon}-a_{\varepsilon} \otimes n_{\varepsilon}\right| \leq C .
$$

Observe that clearly $u_{\varepsilon} \rightarrow u$ in $L^{1}$ and $D u_{\varepsilon} \rightarrow D u$ in the flat norm (even weak* in $\mathcal{M})$. Also note that $\nabla u_{\varepsilon}-a_{\varepsilon} \otimes n_{\varepsilon} \rightarrow \nabla u$ in $L^{2}$. So indeed $\gamma_{\varepsilon}:=a_{\varepsilon} \odot n_{\varepsilon} \rightarrow$ $\frac{1}{2}\left(\left(D^{s} u\right)^{T}+D^{s} u\right)=E^{s} u$.

Choose $\delta=\varepsilon^{2}$ and set $J_{\delta}=J_{\delta}^{1} \cup \cdots \cup J_{\delta}^{m}$. Then the following estimate concludes the proof:

$$
\begin{aligned}
& \limsup _{\varepsilon \rightarrow 0} E_{\varepsilon}\left(u_{\varepsilon}, \gamma_{\varepsilon}\right) \\
& =\limsup _{\varepsilon \rightarrow 0} \int_{\Omega} W\left(\mathcal{E} u_{\varepsilon}-a_{\varepsilon} \odot n_{\varepsilon}\right)+\frac{G}{2 \varepsilon}\left|\left\{a_{\varepsilon} \neq 0\right\}\right| \\
& \leq \limsup _{\varepsilon \rightarrow 0} \int_{\Omega \backslash J_{\delta}} W\left(\mathcal{E} u_{\varepsilon}\right)+\limsup _{\varepsilon \rightarrow 0} \int_{J_{\delta}} W\left(\mathcal{E} u_{\varepsilon}-a_{\varepsilon} \odot n_{\varepsilon}\right)+\limsup _{\varepsilon \rightarrow 0} \frac{G}{2 \varepsilon}\left|J_{\delta+\varepsilon}\right| \\
& \leq \int_{\Omega \backslash J_{u}} W(\mathcal{E} u)+\limsup _{\varepsilon \rightarrow 0} C\left|J_{\delta}\right|+\limsup _{\varepsilon \rightarrow 0} \frac{G}{2 \varepsilon}\left|J_{\delta+\varepsilon}\right| \\
& =\int_{\Omega} W(\mathcal{E} u)+G\left|J_{u}\right| .
\end{aligned}
$$

4.3. The lower bound. Suppose $\left(u_{\varepsilon}, \gamma_{\varepsilon}\right)$ is a sequence in $W^{1,1}\left(\Omega, \mathbb{R}^{d}\right) \times L^{1}\left(\Omega, \mathbb{R}_{\mathrm{sym}}^{d \times d}\right)$ such that $\|u\|_{L^{\infty}} \leq K$ and

$$
E_{\varepsilon}\left(u_{\varepsilon}, \gamma_{\varepsilon}\right) \leq C
$$

If $A$ is an open set contained in $\Omega$, we define the localized energies $E^{A}$ as in the one-dimensional case (see (2.1)) by

$$
E_{\varepsilon}^{A}(u, \gamma)=\int_{A} W(\mathcal{E} u-\gamma)+\frac{G}{2 \varepsilon}\left|\{\gamma \neq 0\}_{\varepsilon} \cap A\right| .
$$

Copyright $@$ by SIAM. Unauthorized reproduction of this article is prohibited. 
Partition $\Omega$ by the regular grid $\frac{\varepsilon}{\sqrt{d}} \mathbb{Z}^{d}$ into sets $Q(y)=\frac{\varepsilon}{\sqrt{d}}\left(y+(0,1)^{d}\right), y \in \mathbb{Z}^{d}$, and define $\tilde{u}_{\varepsilon} \in L^{1}$ by

$$
\tilde{u}_{\varepsilon}(x)= \begin{cases}u_{\varepsilon}(x) & \text { if } \gamma_{\varepsilon}=0 \text { a.e. on } Q(y) \text { and } Q(y) \subset \Omega, \\ 0 & \text { if } \gamma_{\varepsilon} \not \equiv 0 \text { on } Q(y) \text { or } Q(y) \not \subset \Omega\end{cases}
$$

for $x \in Q(y)$. Clearly, $\tilde{u}_{\varepsilon} \in S B D(\Omega)$ with $J_{\tilde{u}_{\varepsilon}} \subset \bigcup_{y \in \mathcal{I}} \partial Q(y)$, where the index set $\mathcal{I}$ collects all $y$ such that $\gamma_{\varepsilon} \not \equiv 0$ on $Q(y)$ and $Q(y) \subset \Omega$.

In case $\gamma_{\varepsilon}=0$ a.e. on $Q(y) \subset \Omega$ we clearly have

$$
E_{\varepsilon}^{Q(y)}\left(u_{\varepsilon}, \gamma_{\varepsilon}\right)=\int_{Q(y)} W\left(\mathcal{E} \tilde{u}_{\varepsilon}\right)
$$

If $\gamma_{\varepsilon}$ does not vanish on $Q(y) \subset \Omega$, then we can estimate

$$
E_{\varepsilon}^{Q(y)}\left(u_{\varepsilon}, \gamma_{\varepsilon}\right) \geq \int_{Q(y)} W\left(\mathcal{E} \tilde{u}_{\varepsilon}\right)+\frac{G}{2 \varepsilon}\left(\frac{\varepsilon}{\sqrt{d}}\right)^{d} .
$$

Summing over all $y$ we find that

$$
C \geq E_{\varepsilon}\left(u_{\varepsilon}, \gamma_{\varepsilon}\right) \geq \int_{\Omega} W\left(\mathcal{E} \tilde{u}_{\varepsilon}\right)+\frac{G}{4 d^{3 / 2}} \mathcal{H}^{d-1}\left(J_{\tilde{u}_{\varepsilon}}\right) .
$$

As $K \geq\left\|u_{\varepsilon}\right\|_{L^{\infty}} \geq\left\|\tilde{u}_{\varepsilon}\right\|_{L^{\infty}}$, we may apply Theorem 3.2 to extract a subsequence (not relabeled) such that $\tilde{u}_{\varepsilon} \rightarrow u$ in $L^{1}$ for some $u \in S B D^{2}(\Omega), \mathcal{E} \tilde{u}_{\varepsilon} \rightarrow \mathcal{E} u$ in $L^{2}$, and

$$
\liminf _{\varepsilon \rightarrow 0} E_{\varepsilon}\left(u_{\varepsilon}, \gamma_{\varepsilon}\right) \geq \int_{\Omega} W(\mathcal{E} u) d x+\frac{G}{4 d^{3 / 2}} \mathcal{H}^{d-1}\left(J_{u}\right) .
$$

Setting $A_{\varepsilon}:=\bigcup_{y \in \mathcal{I}} Q(y) \cup\{x: \operatorname{dist}(x, \partial \Omega) \leq \varepsilon\}$ and using (4.1), we have $\left|A_{\varepsilon}\right| \rightarrow 0$ because $\bigcup_{y \in \mathcal{I}} Q(y) \subset\left\{\gamma_{\varepsilon} \neq 0\right\}_{\varepsilon}$ and $\partial \Omega$ is Lipschitz. In particular, since $u_{\varepsilon}$ and $\tilde{u}_{\varepsilon}$ coincide outside $A_{\varepsilon}$ and are uniformly bounded in $L^{\infty}$, we observe that also

$$
u_{\varepsilon} \rightarrow u \quad \text { in } L^{q} \quad \forall q<\infty .
$$

But then $\mathcal{E} u_{\varepsilon} \rightarrow E u$ in $W^{-1, q}$. Noting that $\chi_{A_{\varepsilon}}$ converges to 0 in measure, that $\mathcal{E} u_{\varepsilon}-\gamma_{\varepsilon}$ is bounded in $L^{2}$ by (4.1) and $\mathcal{E} \tilde{u}_{\varepsilon}$ converges to $\mathcal{E} u$ weakly in $L^{2}$ (as noted above), and that $A_{\varepsilon} \supset\left\{\gamma_{\varepsilon} \neq 0\right\}_{\varepsilon}$, we therefore obtain

$$
\begin{aligned}
\gamma_{\varepsilon} & =\chi_{A_{\varepsilon}} \gamma_{\varepsilon}=\chi_{A_{\varepsilon}}\left(\gamma_{\varepsilon}-\mathcal{E} u_{\varepsilon}\right)-\left(1-\chi_{A_{\varepsilon}}\right) \mathcal{E} \tilde{u}_{\varepsilon}+\mathcal{E} u_{\varepsilon} \\
& \rightarrow 0-\mathcal{E} u+E u=E^{s} u=: \gamma
\end{aligned}
$$

in $W^{-1, q}$ for all $q<2^{*}$ by the Rellich-Kondrachov theorem. So it remains to show that

$$
\liminf _{\varepsilon \rightarrow 0} E_{\varepsilon}\left(u_{\varepsilon}, \gamma_{\varepsilon}\right) \geq E(u) .
$$

Let $\Xi$ be the hyperplane with normal $\xi$. Choose an orthogonal matrix $R$ such that $\Xi=R \mathbb{R}^{d-1}$ and $\xi=R e_{d}$. Let $\rho>0$ and define cubes $Q_{\rho}^{R}(y)=\rho R\left(y+[0,1]^{d}\right)$ for $y \in \mathbb{Z}^{d}$. If $U \subset \Omega$ is some open set, we denote by $U_{\rho}$ the interior of the union of cubes $Q_{\rho}^{R}(y)$ contained in $U$. Note that similarly as above we obtain a localized version of 
(4.3) by replacing $\Omega$ by $U_{\rho}$. Then taking the supremum over all $\rho>0$ shows that in fact

$$
\liminf _{\varepsilon \rightarrow 0} E_{\varepsilon}^{U}\left(u_{\varepsilon}, \gamma_{\varepsilon}\right) \geq \int_{U} W(\mathcal{E} u) d x+\frac{G}{4 d^{3 / 2}} \mathcal{H}^{d-1}\left(J_{u} \cap U\right) .
$$

For a.e. $z \in \Xi$ we have $u_{\varepsilon}^{z, \xi} \in W^{1,2}\left(\Omega^{z, \xi}\right), u_{\varepsilon}^{z, \xi} \rightarrow u^{z, \xi}$ in $L^{1}\left(\Omega^{z, \xi}\right)$ (upon passing to a further subsequence), and $\gamma_{\varepsilon}^{z, \xi} \in L^{1}\left(\Omega^{z, \xi}\right)$, given by $\gamma_{\varepsilon}^{z, \xi}(t)=\xi^{T} \gamma_{\varepsilon}(z+t \xi) \xi$. Since $W$ is positive definite, it is not hard to see that there exists a constant $c>0$ such that

$$
W(A) \geq c\left|\xi^{T} A \xi\right|^{2} \quad \forall A \in \mathbb{R}_{\mathrm{sym}}^{d \times d}, \xi \in S^{d-1}
$$

(resp., $W(a) \geq c|a \cdot \xi|$ for all $a \in \mathbb{R}^{d}$ in the antiplane shear model).

Recall from Theorem 3.1 that $\left(u^{z, \xi}\right)^{\prime}(t)=\xi^{T} \mathcal{E} u(z+t \xi) \xi$ for a.e. $t$. It follows that

$$
\begin{aligned}
C & \geq E_{\varepsilon}^{U_{\rho}}\left(u_{\varepsilon}, \gamma_{\varepsilon}\right) \\
& \geq \int_{\Xi}\left[\int_{U_{\rho}^{z, \xi}} c\left|\left(u_{\varepsilon}^{z, \xi}\right)^{\prime}-\gamma_{\varepsilon}^{z, \xi}\right|^{2} d t+\frac{G}{2 \varepsilon} \mathcal{H}^{1}\left(\left\{\gamma_{\varepsilon}^{z, \xi} \neq 0\right\}_{\varepsilon} \cap U_{\rho}^{z, \xi}\right)\right] d z .
\end{aligned}
$$

By construction each $U_{\rho}^{z, \xi}$ is a finite union of intervals, so the $\lim \inf _{\varepsilon \rightarrow 0}$ of the term $[\cdots]$ in brackets can be estimated using our one-dimensional result in Theorem 2.1 by the limiting one-dimensional energy which is

$$
\int_{U_{\rho}^{z, \xi}} c\left|\left(u^{z, \xi}\right)^{\prime}\right|^{2}+G \#\left(J_{u^{z, \xi}} \cap U_{\rho}^{z, \xi}\right)
$$

for $u^{z, \xi} \in S B V^{2}\left(U_{\rho}^{z, \xi}\right),\left\|u^{z, \xi}\right\|_{L^{\infty}} \leq K$, and which equals $\infty$ otherwise. So by Fatou's lemma and Theorem 3.1

$$
\liminf _{\varepsilon \rightarrow 0} E_{\varepsilon}^{U_{\rho}}\left(u_{\varepsilon}, \gamma_{\varepsilon}\right) \geq \int_{U_{\rho}} c\left|\xi^{T} \mathcal{E} u(x) \xi\right|^{2} d x+G \int_{J_{u} \cap U_{\rho}}\left|n_{u} \cdot \xi\right| d \mathcal{H}^{d-1}
$$

Now letting $\rho \rightarrow 0$ we are led to

$$
\liminf _{\varepsilon \rightarrow 0} E_{\varepsilon}^{U}\left(u_{\varepsilon}, \gamma_{\varepsilon}\right) \geq \int_{U} c\left|\xi^{T} \mathcal{E} u(x) \xi\right|^{2} d x+G \int_{J_{u} \cap U}\left|n_{u} \cdot \xi\right| d \mathcal{H}^{d-1} .
$$

We conclude the proof by noting that according to (4.4)

$$
\liminf _{\varepsilon \rightarrow 0} E_{\varepsilon}^{U}\left(u_{\varepsilon}, \gamma_{\varepsilon}\right) \geq \int_{U} W(\mathcal{E} u) d x
$$

and, due to (4.5),

$$
\liminf _{\varepsilon \rightarrow 0} E_{\varepsilon}^{U}\left(u_{\varepsilon}, \gamma_{\varepsilon}\right) \geq G \int_{J_{u} \cap U}\left|n_{u} \cdot \xi\right| d \mathcal{H}^{d-1} .
$$

An application of Proposition 4.3 with $\mu(U):=\liminf _{\varepsilon \rightarrow 0} E^{U}\left(u_{\varepsilon}, \gamma_{\varepsilon}\right)$ and $\lambda=\mathcal{L}^{d}\lfloor(\Omega \backslash$ $\left.J_{u}\right)+\mathcal{H}^{d-1}\left\lfloor J_{u}\right.$ yields that

$$
\liminf _{\varepsilon \rightarrow 0} E\left(u_{\varepsilon}, \gamma_{\varepsilon}\right) \geq \int_{\Omega} W(\mathcal{E} u) d x+G \int_{J_{u}}\left|n_{u}\right| d \mathcal{H}^{d-1}=E(u, \gamma) .
$$

Proposition 4.3 (cf. [11, Proposition 1.16]). Let $\mathcal{A}$ be the collection of open sets contained in $\Omega$. Suppose $\mu: \mathcal{A} \rightarrow[0, \infty)$ is superadditive on open sets with disjoint compact closures and $\lambda$ is a positive Borel measure on $\Omega$. Let $\psi_{i}$ be positive Borel functions such that $\mu(A) \geq \int_{A} \psi_{i} d \lambda$ for all $A \in \mathcal{A}(\Omega)$. Then $\mu(A) \geq \int_{A} \sup _{i} \psi_{i} d \lambda$ for all $A \in \mathcal{A}(\Omega)$.

Copyright $@$ by SIAM. Unauthorized reproduction of this article is prohibited. 
5. The finite element approximation. Suppose $\mathcal{T}_{h}$ is a family of meshes; i.e., each $\mathcal{T}_{h}$ is a set of simplices with pairwise disjoint interiors that cover all of $\mathbb{R}^{d}$. E.g., $\mathcal{T}_{h}$ could be a family of regular meshes obtained from a periodic grid in $\mathbb{R}^{d}$. However, we do not make any assumptions on the geometry of the cells but suppose only that

$$
\sup \left\{\operatorname{diam} T: T \in \mathcal{T}_{h}\right\}=\mathcal{O}(h) \quad \text { as } \quad h \rightarrow 0 .
$$

By $V_{h}$ and $W_{h}$ we denote the corresponding finite element spaces of piecewise affine functions (which are continuous across element boundaries) and of piecewise constant functions, respectively.

5.1. The static case. We first examine, as in the previous sections, the case in which $\Omega$ is loaded by fixed displacement boundary conditions $u=g$ on the Dirichlet part $\partial \Omega_{D}$ of the boundary $\partial \Omega$, assuming the Neumann part of $\partial \Omega$ to be traction free and body forces to be absent. As before, the inclusion of the latter effects is rather straightforward.

Fix a function $\varepsilon=\varepsilon(h)$ such that $\varepsilon \rightarrow 0$ and $h \varepsilon^{-1} \rightarrow 0$ as $h \rightarrow 0$. For a set $A \subset \Omega$ we define the $\varepsilon$-neighborhood $A_{h, \varepsilon}$ with respect to the mesh $\mathcal{T}_{h}$ as the union of elements $T \in \mathcal{T}_{h}$ such that $\operatorname{dist}(T, A):=\inf \{|x-y|: x \in T, y \in A\} \leq \varepsilon$. For $u \in L^{1}\left(\Omega, \mathbb{R}^{d}\right)$ and $\gamma \in \mathcal{M}\left(\Omega, \mathbb{R}_{\mathrm{sym}}^{d \times d}\right)$ let

$$
\tilde{E}_{h}(u, \gamma):= \begin{cases}\int_{\Omega} W(\mathcal{E} u-\gamma)+\frac{G}{2 \varepsilon}\left|\{\gamma \neq 0\}_{h, \varepsilon(h)}\right| & \text { if } u \in V_{h},\|u\|_{L^{\infty}} \leq K, \\ & \gamma \in W_{h}, \\ \infty & \text { otherwise. }\end{cases}
$$

The limiting functional $E: L^{1}\left(\Omega, \mathbb{R}^{d}\right) \times \mathcal{M}\left(\Omega, \mathbb{R}_{\mathrm{sym}}^{d \times d}\right) \rightarrow \mathbb{R} \cup\{\infty\}$ is as defined before.

The following theorem is basically a corollary to Theorem 4.1 (and its proof). So we merely indicate the necessary changes and additional arguments.

TheOREM 5.1 ( $\Gamma$-convergence and compactness). Let $L^{1}\left(\Omega, \mathbb{R}^{d}\right)$ be equipped with the strong topology and $\mathcal{M}\left(\Omega, \mathbb{R}_{\mathrm{sym}}^{d \times d}\right)$ with the flat topology. Then $\tilde{E}_{h} \Gamma$-converges to $E$ on $L^{1}\left(\Omega, \mathbb{R}^{d}\right) \times \mathcal{M}\left(\Omega, \mathbb{R}_{\mathrm{sym}}^{d \times d}\right)$. Furthermore, bounded energy sequences are compact in this topology and in $L^{1}\left(\Omega, \mathbb{R}^{d}\right) \times W^{-1, q}$ for all $q<2^{*}$. Moreover, the recovery sequence $\left(u_{h}, \gamma_{h}\right)$ can be chosen such that $\gamma_{h}=a_{h} \odot n_{h}$ for suitable piecewise constant $a_{h}, n_{h}$ with $\left|n_{h}\right|=1$.

Proof. First note that $V_{h} \subset W^{1,1}$ and $W_{h} \subset L^{1}$. Since

$$
\tilde{E}_{h}(u, \gamma) \geq E_{\varepsilon}(u, \gamma)
$$

for all $u \in V_{h}, \gamma \in W_{h}$, the lim inf-inequality and the compactness of bounded energy sequences are immediate.

To provide a recovery sequence for $u \in \mathcal{W}\left(\Omega, \mathbb{R}^{d}\right)$ we first let $\tilde{u}_{\varepsilon}=\tilde{u}_{\varepsilon(h)}$ be the function constructed in section 4.2 (denoted $u_{\varepsilon}$ there) but with $\delta=\delta(h)$ such that $h \ll \delta \ll \varepsilon$. Now define $u_{h} \in V_{h}$ to be the piecewise affine interpolation of $\tilde{u}_{\varepsilon(h)}$ with respect to $\mathcal{T}_{h}$. From the regularity properties of $u$ on $\Omega \backslash J_{u}$ it is not hard to deduce that we can choose $a_{h} \in W_{h}$, vanishing outside the $(\delta+h)$-neighborhood of $J_{u}$ such that again

$$
\left|\nabla u_{h}-a_{h} \otimes n_{h}\right| \leq C,
$$

where $n$ is defined as before. Moreover, the regularity of $u$ away from its jump set guarantees that $u_{h} \rightarrow u$ in $W^{1,2}$ away from $J_{u}$. The remaining part of the proof of (ii) is analogous to the proof in section 4.2 . 
Remarks.

(i) In our numerical implementation it is more convenient to measure the distance between two sets $A=\bigcup_{i \in \mathcal{I}_{1}} T_{i}$ and $B=\bigcup_{i \in \mathcal{I}_{2}} T_{i}, T_{i} \in \mathcal{T}_{h}$, by evaluating the distance between specific points $x_{i} \in T_{i}$, e.g., the barycenters of $T_{i}$, setting $\operatorname{dist}(A, B):=\inf \left\{\left|x_{i_{1}}-x_{i_{2}}\right|: i_{1} \in \mathcal{I}_{1}, i_{2} \in \mathcal{I}_{2}\right\}$. This leads of course to the same results; one simply has to note that

$$
\tilde{E}_{h}(u, \gamma) \geq \int_{\Omega} W(\mathcal{E} u-\gamma)+\frac{G}{2 \varepsilon}\left|\{\gamma \neq 0\}_{\varepsilon(h)-h}\right| \geq \frac{\varepsilon-h}{\varepsilon} E_{\varepsilon-h}(u, \gamma) .
$$

(ii) Again it is straightforward to include boundary values on the Dirichlet boundary $\partial \Omega_{D}=\tilde{\Omega} \cap \partial \Omega$. Assuming that $g \in W^{2, \infty}\left(\tilde{\Omega}, \mathbb{R}^{d}\right)$ with $\|g\|_{L^{\infty}} \leq K$, in the finite element approximation this can be achieved by requiring that, for $u \in V_{h}, u(x)=g(x)$ for all nodal points $x$ of the triangulation $\mathcal{T}_{h}$ that lie in $\tilde{\Omega} \backslash \Omega$. Note that in fact in the numerical examples investigated in section 6 we do not need to extend our domain $\Omega$ to the larger domain $\tilde{\Omega}$ since our Dirichlet boundaries $\partial \Omega_{D}$ are perfectly aligned with the edges/faces of $\mathcal{T}_{h}$ for each $h$.

The finite element counterpart of Theorem 4.2 approximates the jump set of the continuum displacement field by jumps across element boundaries. We discretize $F_{\varepsilon}$ by restricting to the finite element space $X_{h} \subset S B D^{2}(\Omega)$ of piecewise affine functions (which are not necessarily smooth across element boundaries). Fix a function $\varepsilon=\varepsilon(h)$ such that $\varepsilon \rightarrow 0$ and $h \varepsilon^{-1} \rightarrow 0$ as $h \rightarrow 0$. Now consider the approximations

$$
\tilde{F}_{h}(u):= \begin{cases}\int_{\Omega} W(\mathcal{E} u)+\frac{G}{2 \varepsilon}\left|\left(J_{u}\right)_{\varepsilon, h}\right| & \text { if } u \in X_{h}(\Omega),\|u\|_{L^{\infty}} \leq K \\ \infty & \text { otherwise. }\end{cases}
$$

As a corollary to Theorem 4.2 we obtain the following.

THEOREM 5.2 ( $\Gamma$-convergence and compactness of finite elements). $\tilde{F}_{h} \Gamma$-converges to $F$ with respect to the $L^{2}\left(\Omega, \mathbb{R}^{d}\right)$-metric. Furthermore, bounded energy sequences are compact in this topology.

5.2. Quasi-static evolution. Next we consider a quasi-static evolution problem governed by a displacement load history $u=\lambda(t) \bar{u}(x)$ on $\partial \Omega_{D}, \lambda(t)$ being a prescribed loading function, and $t \in[0, T]$. Let $0<t_{1}<\cdots<t_{N}=T$ be a time discretization of the problem and $V_{h}^{(j)}$ the finite element set of piecewise linear functions $u$ such that $u=\lambda\left(t_{j}\right) \bar{u}(x)$ on $\partial \Omega_{D}$. Furthermore, let $\tilde{E}_{h}^{(j)}$ be the functional defined as $\tilde{E}_{h}$ in (5.1) under the replacement of $V_{h}$ with $V_{h}^{(j)}$, and $K_{h}^{(j)}$ the set $\left\{\gamma_{h}^{(j)} \neq 0\right\}$. For the sake of notational simplicity, the superscript $(j)$ is henceforth dropped.

The recovery sequences constructed in the proofs of Theorems 4.1 and 5.1 satisfy $\gamma=a \odot n$ and $\gamma_{h}=a_{h} \odot n_{h}$, respectively, but nevertheless the above theorems show that minimizing over arbitrary $\gamma \in \mathcal{M}\left(\Omega, \mathbb{R}_{\text {sym }}^{d \times d}\right)$ and $\gamma_{h} \in W_{h}$ eventually give the same results if $h$ is small. We name full rank (FRM) the finite element model that lets $\gamma_{h}$ be free in $W_{h}$, and rank one (ROM) the model instead assuming $\gamma_{h}=a_{h} \odot n_{h}$, for piecewise constant $a_{h}, n_{h}\left(\left|n_{h}\right|=1\right)$.

Looking at the structure of the above recovery sequences, we adopt a particular choice of $\gamma_{h}$, which proves to be helpful for the numerical implementation of the eigenfracture model, and accordingly reformulate the discrete problem. In detail, we assume $\gamma_{h}:=\left(\nabla u_{h} n_{h}\right) \odot n_{h}$ on $K_{h}$ and zero outside for the ROM, regarding $u_{h}$, $n_{h}$, and $K_{h}$ as the arguments of the discrete energy functional $\hat{E}_{h}\left(u_{h}, n_{h}, K_{h}\right):=$ $\tilde{E}_{h}\left(u_{h}, \gamma_{h}\left(u_{h}, n_{h}, K_{h}\right)\right)$. Similarly, we assume $\gamma_{h}:=\nabla u_{h}$ on $K_{h}$ and zero outside for 
the FRM, regarding $u_{h}$ and $K_{h}$ as the arguments of $\hat{E}_{h}\left(u_{h}, K_{h}\right):=\tilde{E}_{h}\left(u_{h}, \gamma_{h}\left(u_{h}, K_{h}\right)\right)$. The above assumptions turn out to coincide for the recovery sequences $u_{h}, \gamma_{h}$ constructed in section 4.2 as $h \rightarrow 0$, when $\nabla u_{h} \approx a_{h} \otimes n_{h}$, and $\left(\nabla u_{h}\right)^{T} \nabla u_{h} \approx\left|a_{h}\right|^{2} n_{h} \odot$ $n_{h}$ on $K_{h}$, for some $a_{h}, n_{h}$. We shortly write $\gamma_{h}:=\hat{\gamma}\left(u_{h}, n_{h}, K_{h}\right)$, and $E_{h}:=$ $\hat{E}_{h}\left(u_{h}, n_{h}, K_{h}\right)$, intending that $n_{h}$ drops for the FRM.

Finite element solutions $u_{h}, n_{h}, K_{h}$ of the discrete problem are determined through an iterative energy-descent procedure, which initially assumes that $n_{h}$ and $K_{h}$ remain unchanged with respect to the previous load step. Subsequently, the procedure predicts a number $P$ of virtual extensions $\Delta K_{p}$ of $K_{h}$, determining the corresponding displacement fields $u_{p}$ and and normals $n_{p}$ through minimization of $\hat{E}_{h}\left(\cdot, \cdot, K_{h} \cup \Delta K_{p}\right)$. If the least of the predictor energies $\hat{E}_{h}\left(u_{p}, n_{p}, K_{h} \cup \Delta K_{p}\right)$ is lower than $\hat{E}_{h}\left(u_{h}, n_{h}, K_{h}\right)$, we assume the corresponding triplet $u_{p}, n_{p}, K_{h} \cup \Delta K_{p}$ as the new guess of the solution $u_{h}, n_{h}, K_{h}$ for the current load step and reiterate the virtual crack prediction. The crack extensions are predicted using an affine $d$-chain $K_{h}^{*}$ [41] ( $d$ indicating the dimension of the problem under consideration), composed of $S$ selected mesh elements $\left\{\Omega^{* 1}, \ldots, \Omega^{* S}\right\}$ that we name crack generators. The predictors $\Delta K_{p}$ are allowed to coincide with affine $d$-chains composed of $C$ mesh elements branching off from $K_{h}^{*}$ according to a predefinite pattern (say, e.g., forming a linear collection of mesh simplices). One can think of $K_{h}$ and $K_{h}^{*}$ as the current cracked region and crack front, respectively. However, to be more general, it is useful to distinguish the following two cases: (a) $K_{h}$ is empty at time zero; (b) $K_{h}$ collects a set of predamaged elements $\left(\gamma_{h} \neq 0\right)$ at time zero, simulating a precrack in the body (in the first case, a precrack might instead be modeled as a portion of the boundary $\partial \Omega$ ). For the case of (a), we identify $K_{h}^{*}$ at time zero with the set of mesh simplices where the $S$ th-largest eigenvalues of $\left(\nabla u_{h}\right)^{T} \nabla u_{h}$ are attained. Furthermore, in the same case, we let $\Delta K_{p}$ be either a single simplex or a cluster of simplices belonging to the union of $K_{h}^{*} \backslash\left(K_{h}^{*} \cap K_{h}\right)$ and the star of $\partial K_{h}^{*}$ (with respect to the mesh $\mathcal{T}_{h}$ ). For the case of (b), we instead identify $K_{h}^{*}$ at time zero with the subset of $K_{h}$ describing the crack front and let $\Delta K_{p}$ be either a single simplex or a cluster of simplices belonging to the star of $\partial K_{h}^{*}$. In each case, a generic virtual crack extension $\Delta K_{p}$ is generated from a simplex $\Omega^{* s} \in K_{h}^{*}$, being directly an element of such a set or a $d$-chain attached to an element of $K_{h}^{*}$. Once a $\Delta K_{p}$ is added to the current crack $K_{h}$, the corresponding $\Omega^{* s}$ in $K_{h}^{*}$ is replaced with the leading element of $\Delta K_{p}$.

It is worth noting that the proposed solution search strategy allows for modeling an arbitrary crack pattern in any dimension, including, e.g., multiple branched cracks, under the condition that the size $S$ of $K_{h}^{*}$ is sufficiently large. Due to the recursive exploration of virtual crack extensions, $K_{h}$ can be augmented with any number of mesh simplices during each time step. Nevertheless, the search of the current crack is obviously influenced by the particular choice made for $S, P$, and $C$, and thus a convergence study on each of such parameters should be preliminarily performed, searching for sets of parameters that ensure stable predictions. One could assume $P=(d+1) S$, fitting $S$ and $C$ to the problem on hand through numerical experiments. We summarize the main steps of the solution strategy in Algorithms 1 and 2 that follow, observing that it can be read as an energy-descent element erosion technique [47]. Indeed, due to the particular choice made for $\gamma_{h}$, the elements of $K_{h}$ are either completely inert (FRM), or behave as partially reactive elastic elements, with zero stiffness in the direction of $n_{h}(\mathrm{ROM})$. The proposed strategy differs from a classical element erosion-type method because it is based on an energy-descent criterion, while standard erosion methods adopt local stress-strain criteria (cf., e.g., [1]). We 


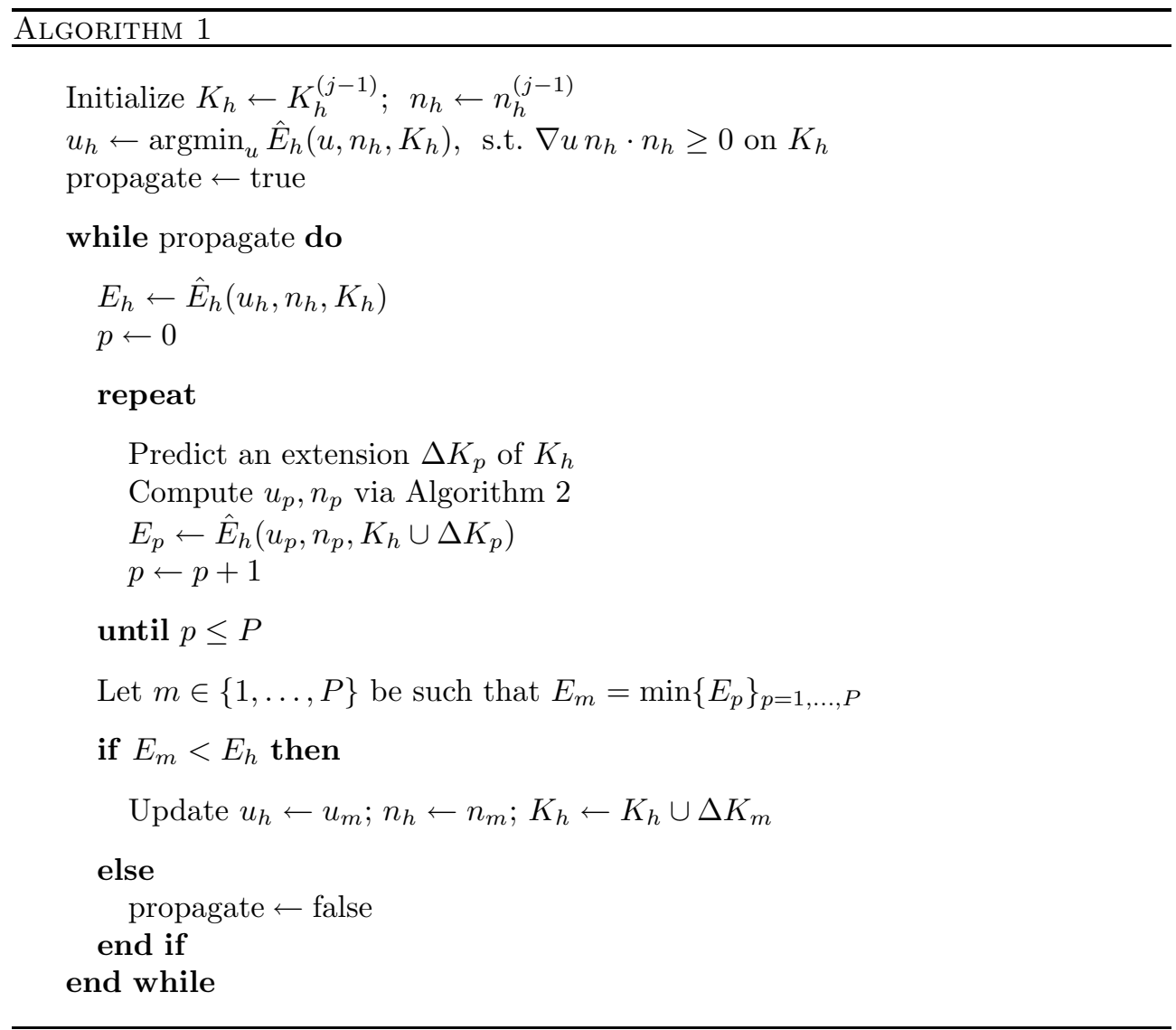

ALGORITHM 2

Initialize $u_{p} \leftarrow u_{h} ; n_{p} \leftarrow n_{h}$

repeat

$u_{p^{\prime}} \leftarrow \operatorname{argmin}_{u} \hat{E}_{h}\left(u, n_{p}, K_{h} \cup \Delta K_{p}\right)$, s.t. $\nabla u n_{p} \cdot n_{p} \geq 0$ on $K_{h} \cup \Delta K_{p}$ Compute $n_{p^{\prime}}$ on $\Delta K_{p}$ as the eigenvector associated with the largest positive eigenvalue of $\left(\nabla u_{p^{\prime}}\right)^{T} \nabla u_{p^{\prime}}$

$n_{p^{\prime}} \leftarrow n_{h}$ on $K_{h}$

$\Delta n \leftarrow\left\|n_{p^{\prime}}-n_{p}\right\|_{L^{\infty}}$

until $\Delta n>T O L$

enforced the constraint $\nabla u_{h} n_{h} \cdot n_{h} \geq 0$ on $K_{h}$ through a penalty technique. The fracture term of the discrete functional $\hat{E}_{h}\left(u_{h}, n_{h}, K_{h}\right)$ depends on the measure of the $\varepsilon$-neighborhood $K_{h, \varepsilon(h)}$ of $K_{h}$ with respect to the mesh $\mathcal{T}_{h}$. This proves to be useful to avoid mesh anisotropy effects and overestimation of fracture energy, which are common drawbacks of fixed grid approaches (cf., e.g., [43]), as demonstrated by the $\Gamma$-convergence result of Theorem 5.1. By way of example, consider a sequence $K_{h}$ that zig-zag converges to the jump set $J_{u}$ of a limit displacement field $u$ as $h \rightarrow 0$.

Copyright (c) by SIAM. Unauthorized reproduction of this article is prohibited. 

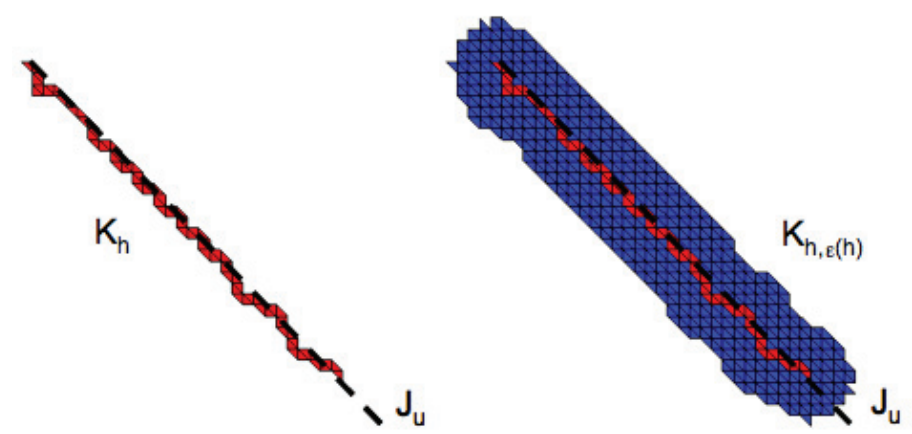

FIG. 5.1. Transverse view of the $\varepsilon$-neighborhood $K_{h, \varepsilon(h)}$ (right) of a discrete crack set $K_{h}$ (left) that zig-zag converges to a planar crack surface.

The measure of the middle surface of $K_{h}$ does not converge to $\left|J_{u}\right|$, while the quantity $\frac{1}{2 \varepsilon}\left|K_{h, \varepsilon(h)}\right|$ does converge to $\left|J_{u}\right|$ (Figure 5.1).

A $\Gamma$-convergence proof of the quasi-static evolution as, e.g., studied by Giacomini in [35] for antiplane shear in the Ambrosio-Tortorelli approximation scheme is beyond the scope of the present study.

6. Numerical results. Simulations of brittle crack propagation under combined I-II and I-III modes were conducted with reference to a prismatic plate with a throughthe-thickness edge precrack (Figure 6.1). The plate is loaded by uniform distributions of displacements $\bar{u}_{1}, \bar{u}_{2}, \bar{u}_{3}$ on the top and bottom faces, opposite in sign with respect to each other. All the examined problems refer to abstract units. In particular, we assume $H:=H_{1}=H_{2}=1$ and consider the isotropic strain energy density

$$
W(\mathcal{E} u)=\frac{\hat{\lambda}}{2}|\operatorname{tr}(\mathcal{E} u)|^{2}+\hat{\mu}|\mathcal{E} u|^{2}
$$

where $\hat{\lambda}$ and $\hat{\mu}$ are the Lamé coefficients, set equal to 0.8 and 0.4 , respectively.

6.1. Mixed modes I-II. Crack propagation in brittle solids under mixed mode I-II loading has been investigated by a number of authors on the basis of different energetic and stress-based criteria of linear elasticity (cf., e.g., Erdogan and Sih [29]; Cotterell [22]; Cotterel and Rice [23]; Sih [50], [51]). The available approaches predict slightly different kinking angles and that, after kinking, the trajectory of the crack is always such that the mode II stress intensity factor $K_{\text {II }}$ is zero at the tip. Experimental results have also shown that plane cracks after kinking tend to grow perpendicularly to the maximum principal stress (Radon, Lever, and Culver [49]). Here we apply the eigendeformation fracture model to the prediction of kinking and growth of the crack in Figure 6.1, for $\bar{u}_{3}=0$, an initial crack length of $0.25 H_{1}$, and different loading angles $\alpha=\arctan \left(\bar{u}_{2} / \bar{u}_{1}\right)$. Two-dimensional finite element simulations were conducted employing regular triangulations supported by uniform grids of nodes, for different mesh sizes $h$ and penalty parameters $\varepsilon$, assuming either $h=\varepsilon / 2$ or $h=\varepsilon / 4$. Unless otherwise specified, the plate thickness was set equal to $h$. The precrack was modeled through boundary surfaces in unilateral contact. We ran several preliminary tests on families of meshes $\mathcal{T}_{h}$ with mesh sizes $h=0.0125$ (6581 nodes and 12800 elements) and $h=0.00625$ (25961 nodes and 51200 elements), which, respectively, correspond to relatively coarse and fine discretizations of the plate. Such tests led us 


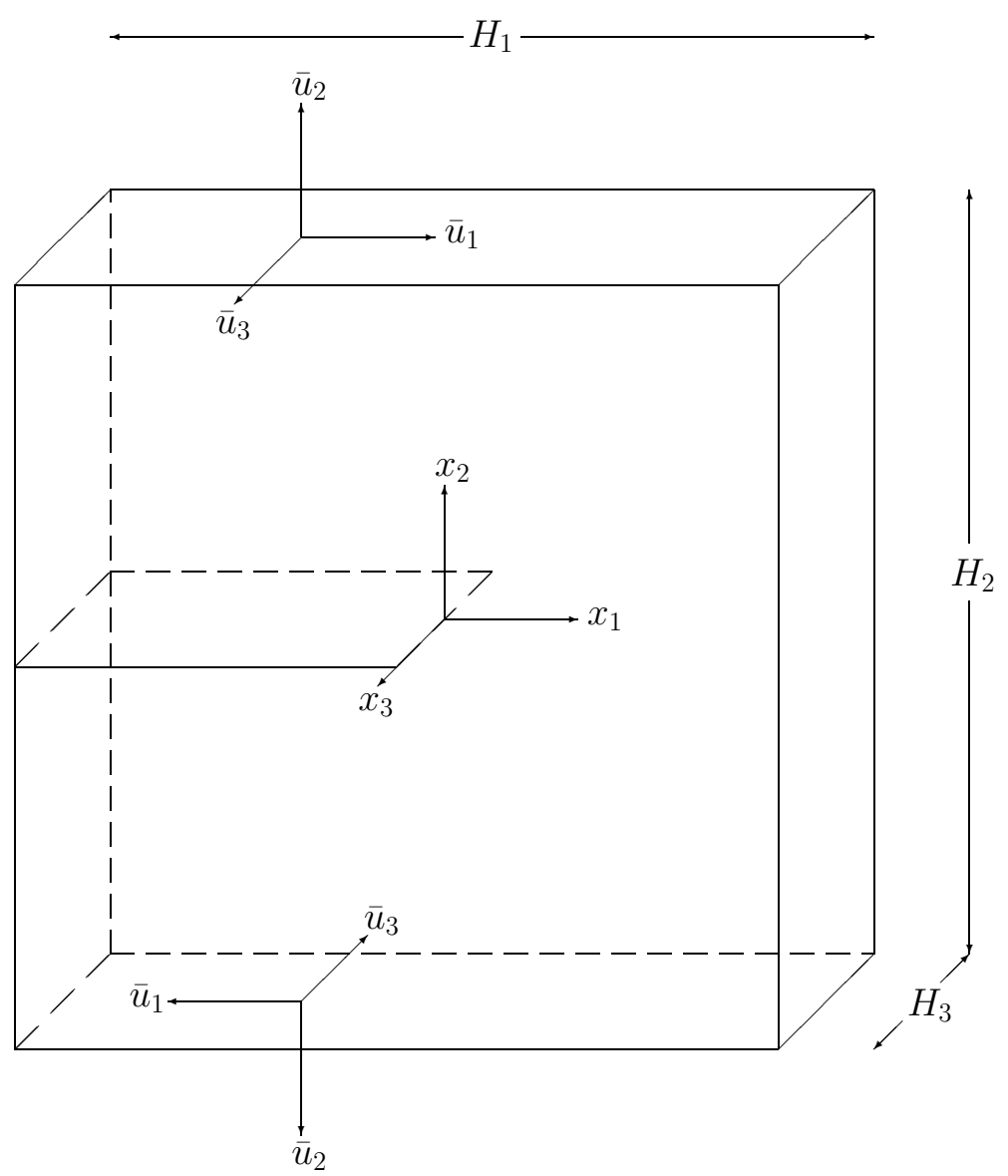

FIG. 6.1. Loaded specimen.

to conclude that reliable crack predictions can be obtained assuming $S=1, P=3$, $C=1, \varepsilon=2 h$ for $h=0.0125$, and $S=2, P=6, C=1, \varepsilon=4 h$ for $h=0.00625$ (virtual crack extensions composed of a single mesh element). The above parameter sets indeed ensured optimal correspondence of the present crack predictions with the results obtained by Bourdin, Francfort, and Marigo in [10] through an AmbrosioTortorelli scheme, among all the other examined values, which included the case with $h=\varepsilon$. In particular, no significant changes of the crack path were observed by slightly reducing the adopted $h / \varepsilon$ ratios (i.e., for $\varepsilon>2 h$ and $\varepsilon>4 h$, respectively), despite the fact that the $\Gamma$-convergence proof of section 5 assumes $h \ll \varepsilon$.

The results corresponding to the ROM are shown in Figures 6.2 and 6.3 for a variety of loading angles and $G=10^{-6}$. Figure 6.2 displays the deformed shapes of the cracked plates (displacement magnification factor $=3$ ), while Figure 6.3 shows the predicted crack paths $K_{h}$ in the reference configuration (in red) and their $\varepsilon$ neighborhoods $K_{h, \varepsilon(h)}$ (in blue). A good agreement between the present results and those given in [10] can be observed. For $\alpha=0$ (mode II), we recorded initial subvertical crack kinking, followed by crack growth approximately at -45 deg (Figure 6.2, $\alpha=0, h=\varepsilon / 4=0.00625)$. This outcome is consistent with the predictions of the maximum hoop stress criterion (initial kinking angle $\theta=-70.6 \mathrm{deg}$ ), the max- 


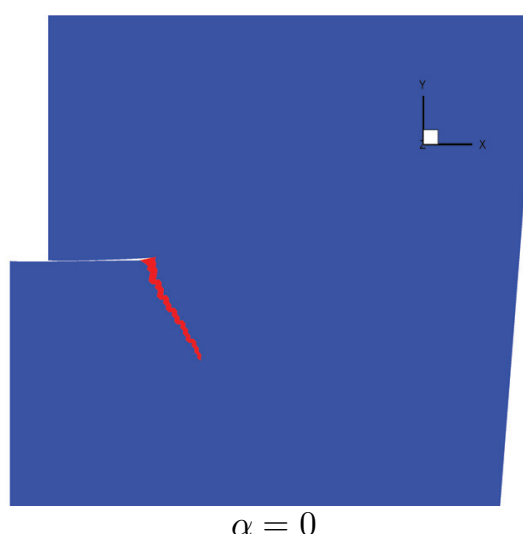

$\alpha=0$

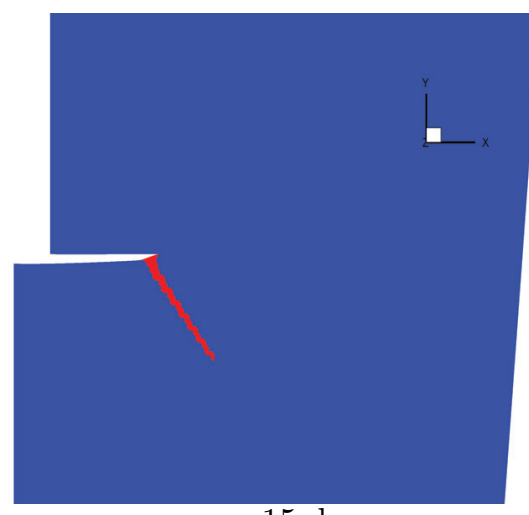

$\alpha=15 \mathrm{deg}$

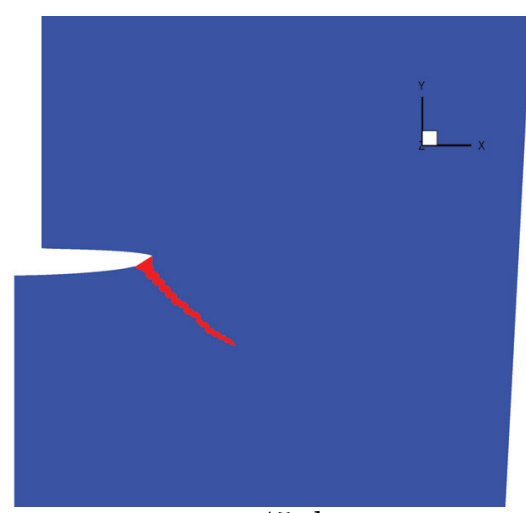

$\alpha=45 \operatorname{deg}$
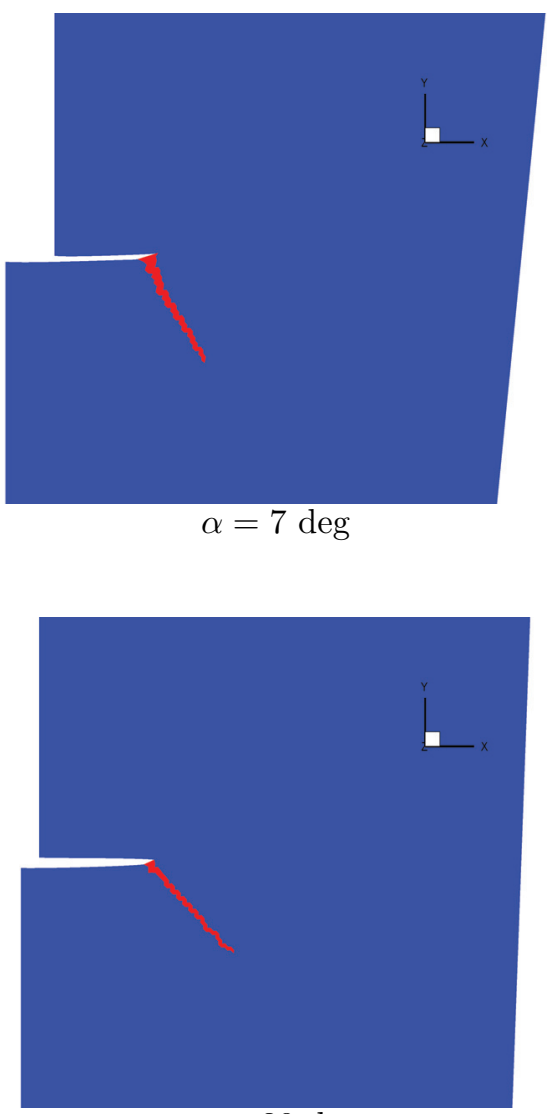

$\alpha=30 \mathrm{deg}$

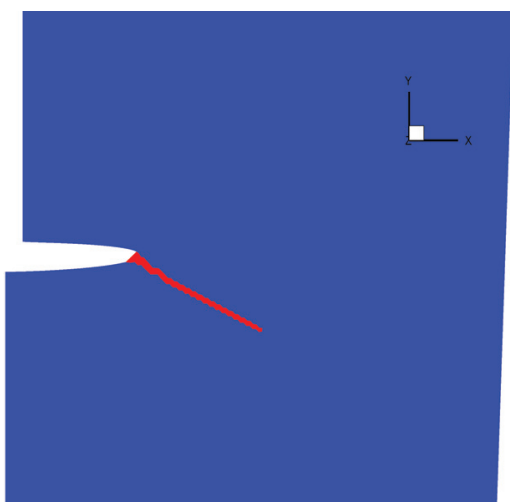

$\alpha=60 \operatorname{deg}$

FIG. 6.2. Deformed shapes for $h=\varepsilon / 4=0.00625$ and different loading angles.

imum energy release rate criterion $(\theta=-75.6 \mathrm{deg})$, and the circumstance that in pure mode II the direction of maximum principal stress is 45 deg to the crack line. The results in Figure 6.3 show that the $\varepsilon$-neighborhood technique is able to correct mesh anisotropy when $h=\varepsilon / 4=0.00625$. Oscillations of the crack path, visible in 

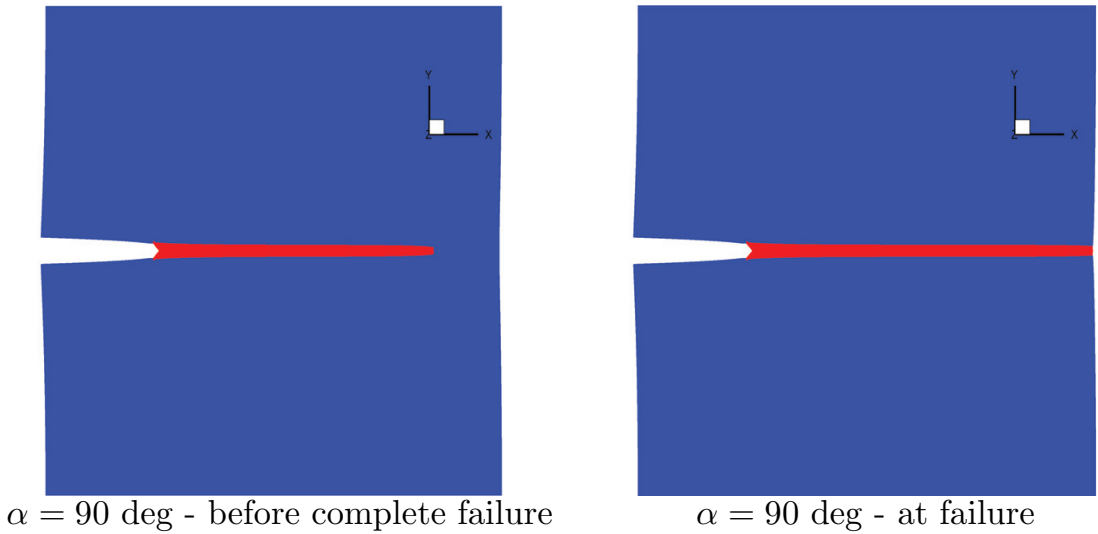

$\alpha=90$ deg - at failure

FIG. 6.2. (cont.).
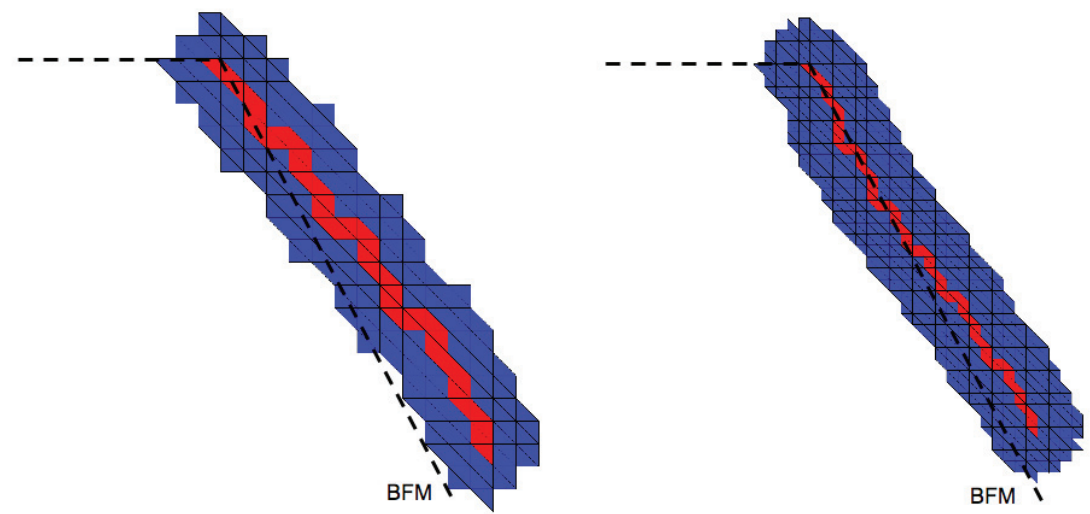

$\alpha=0, h=\varepsilon / 2=0.0125$

$\alpha=0, h=\varepsilon / 4=0.00625$
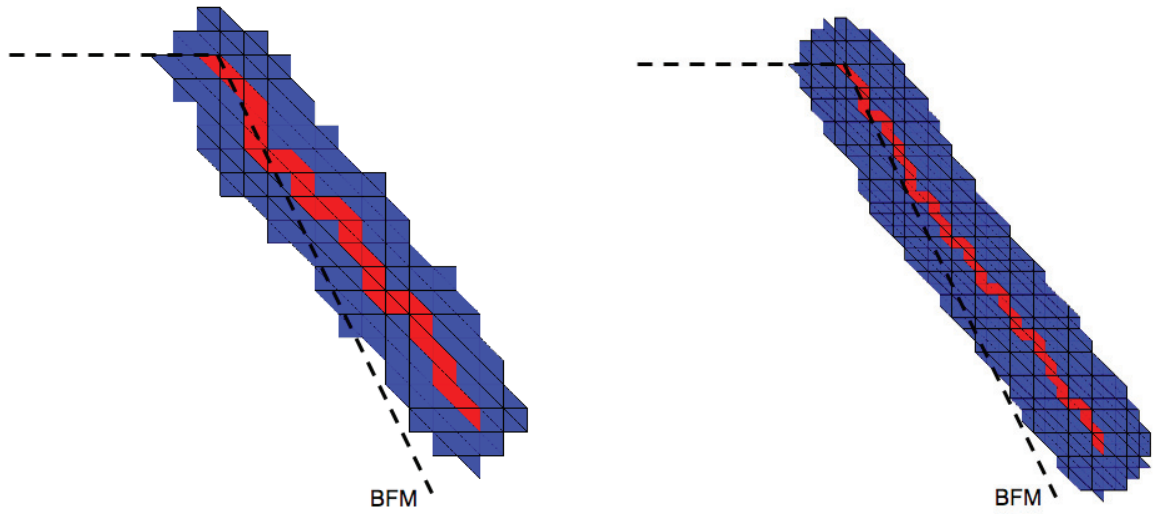

$\alpha=7 \mathrm{deg}, h=\varepsilon / 2=0.0125$

$\alpha=7 \mathrm{deg}, h=\varepsilon / 4=0.00625$

FIG. 6.3. Comparison between current crack path predictions for mixed I-II modes considering different loading angles, mesh sizes, and $\varepsilon$-neighborhoods, and results by Bourdin, Francfort, and Marigo [10]. 


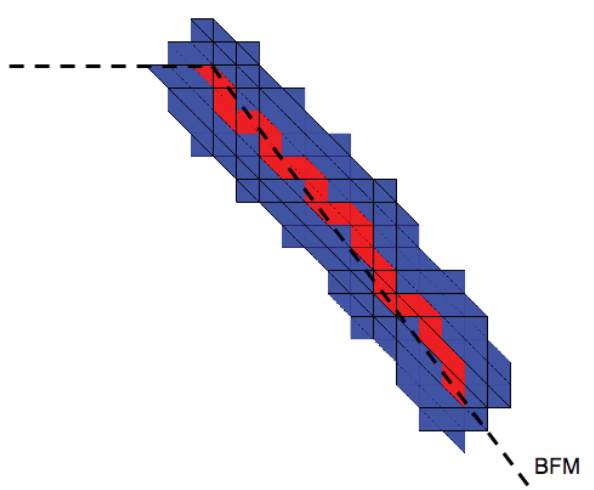

$\alpha=15 \mathrm{deg}, h=\varepsilon / 2=0.0125$

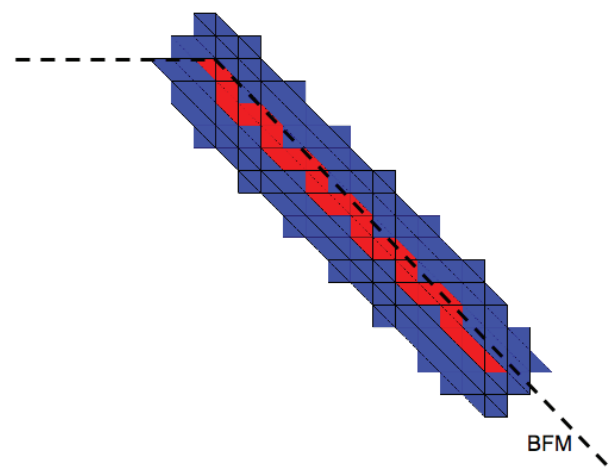

$\alpha=30 \mathrm{deg}, h=\varepsilon / 2=0.0125$

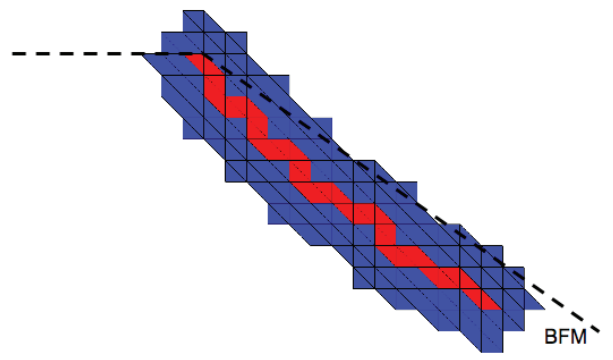

$\alpha=45 \mathrm{deg}, h=\varepsilon / 2=0.0125$

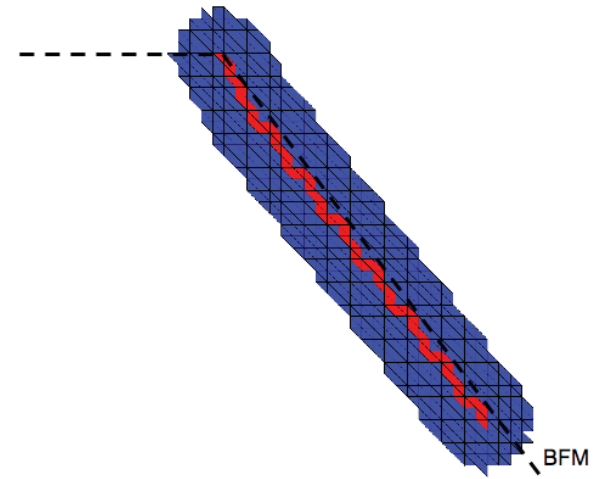

$\alpha=15 \mathrm{deg}, h=\varepsilon / 4=0.00625$

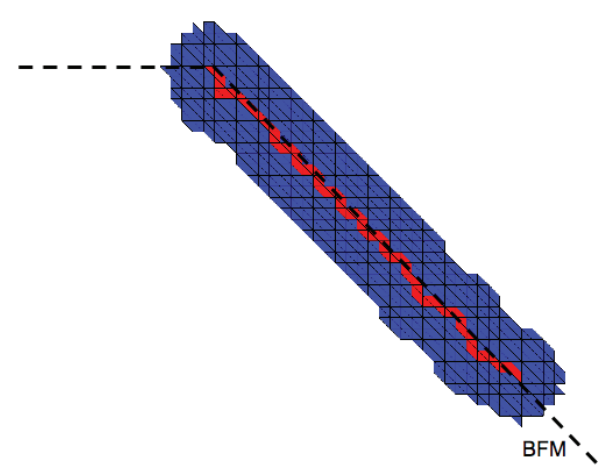

$\alpha=30 \mathrm{deg}, h=\varepsilon / 4=0.00625$

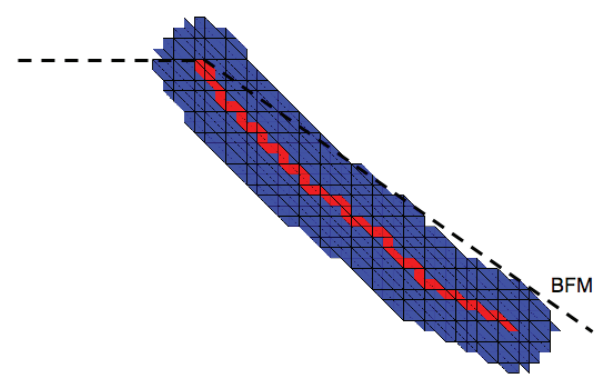

$\alpha=45 \mathrm{deg}, h=\varepsilon / 4=0.00625$

FIG. 6.3. (cont.).

Figure 6.3, are expected to smooth out in the limit $h \rightarrow 0$. For $\alpha=90$ deg (mode I), we modeled one half of the plate and accordingly assumed $G=1 / 2$. The full-body pictures shown in Figures 6.2 and 6.3 for such a case were obtained by mirroring finite element solutions with respect to the symmetry axis. It is worth noticing that the examined loading angles determine kinking angles varying from subvertical to horizontal and not always coinciding with the principal directions $(0,45,90 \mathrm{deg})$ of the employed meshes (cf. [10] and Figure 6.3). 


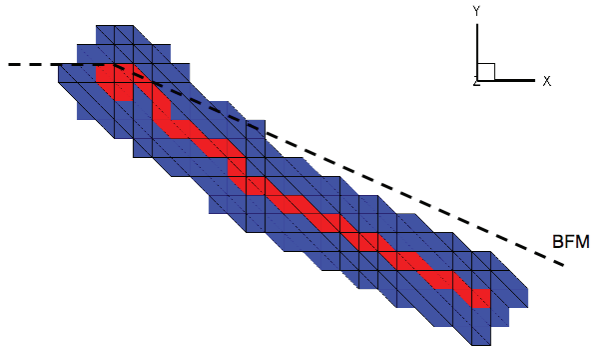

$\alpha=60 \operatorname{deg}, h=\varepsilon / 2=0.0125$

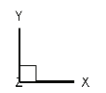

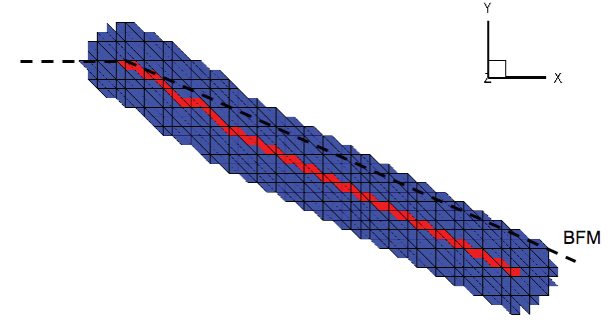

$\alpha=60 \mathrm{deg}, h=\varepsilon / 4=0.00625$

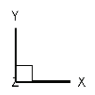

$\alpha=90 \mathrm{deg}, h=\varepsilon / 2=0.0125$

$\alpha=90 \mathrm{deg}, h=\varepsilon / 4=0.00625$

FIG. 6.3. (cont.).

A more detailed convergence study was conducted for $\alpha=45 \mathrm{deg}$ and $\alpha=60 \mathrm{deg}$ considering a third and more refined mesh, with $h=\varepsilon / 4=0.00417$, and $S=2, P=6$, $C=2$ (virtual crack extensions formed by couples of adjacent mesh simplices). We compare in Figure 6.4 the ROM crack path predictions for the above mesh with those obtained through the same approach for $h=\varepsilon / 4=0.00625$. One can observe a good "global" matching of the corresponding $K_{h}$ sets, and the tendency of $K_{h}$ to reproduce the kinking angles given in [10] for the analyzed loading angles, as the mesh size decreases.

The predictions of the ROM and FRM models were compared to each other with reference to $\alpha=45 \mathrm{deg}, G=10^{-4}, \bar{u}_{1}=\bar{u}_{2}=0.707 \times 10^{-4}$, and discretizations with $h=\varepsilon / 4=0.00625$ (a) and $h=\varepsilon / 4=0.00417$ (b). We prescribed $H_{3}=0.00625$ in both cases. The deformed shape and the crack path corresponding to the FRM for $h=\varepsilon / 4=0.00625$ are shown in Figure 6.5. It is seen that such a model predicts crack kinking at $-45 \mathrm{deg}$, straight crack growth, and a second kinking when the tip gets close to the boundary. The first kinking appears for $\lambda=1.60$ and is accompanied by instantaneous crack growth, until the second kinking occurs. After the second kinking, the plate shows hardening behavior, as illustrated by the energy and applied load vs. $\lambda$ plots of Figure 6.6. Here, $\bar{U}_{h}$ and $\bar{V}_{h}$ denote the normalized elastic and fracture components of $E_{h}$, defined as follows:

$$
\bar{U}_{h}=\frac{1}{G H^{2}} \int_{\Omega} W\left(\mathcal{E} u_{h}-\hat{\gamma}_{h}\left(u_{h}, n_{h}, K_{h}\right)\right), \quad \bar{V}_{h}=\frac{1}{2 \varepsilon H^{2}}\left|K_{h, \varepsilon(h)}\right| .
$$

In the same figure, $\bar{E}_{h}$ denotes the sum $\bar{U}_{h}+\bar{V}_{h}$, while $\bar{P}_{h}$ denotes the normalized generalized force defined as

$$
\bar{P}_{h}=\frac{10^{3}}{G H} \int_{\omega} t \cdot \bar{u},
$$




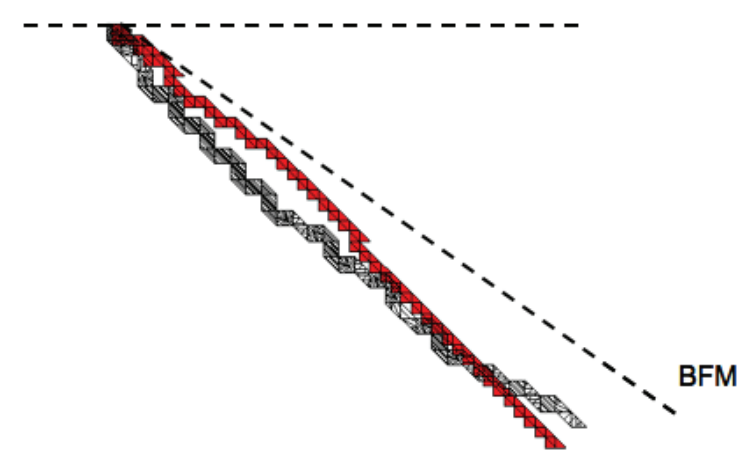

$\alpha=45 \operatorname{deg}$

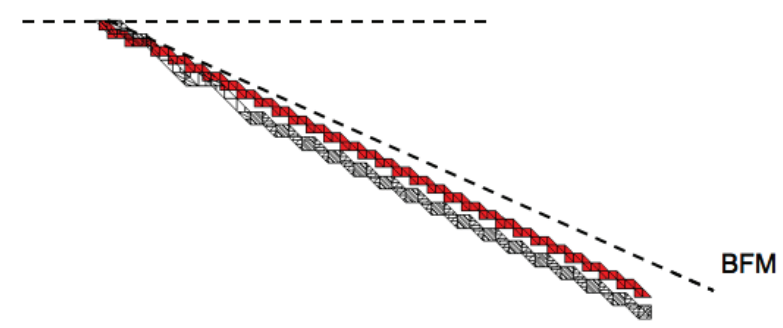

$\alpha=60 \mathrm{deg}$

FIG. 6.4. Comparison between current crack path predictions for $\alpha=45 \mathrm{deg}, \alpha=60$ deg, $h=\varepsilon / 4=0.00625$ (cross-hatched regions), $h=\varepsilon / 4=0.00417$ (red, solid-hatched regions), and the kinking angles predicted by Bourdin, Francfort, and Marigo in [10].
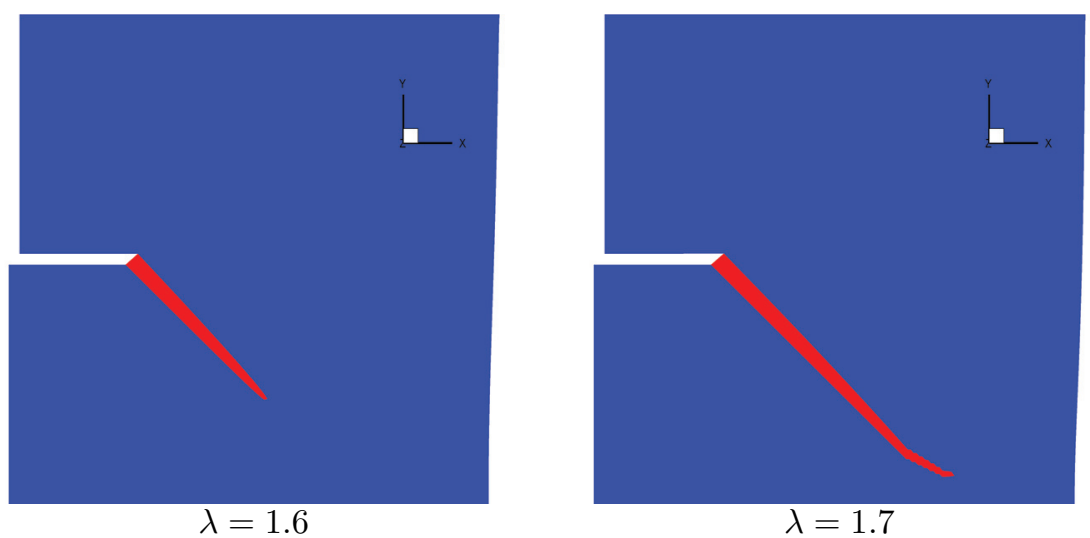

Fig. 6.5. Deformed shapes corresponding to the full rank eigendeformation model for $\alpha=$ $45 \mathrm{deg}, h=\varepsilon / 4=0.00625$.

where $\omega$ is either the top or the bottom face of the plate, $\bar{u}$ is the shape function of the prescribed displacement, and $t$ is the surface traction exerted by the grip constraints. For $h=\varepsilon / 4=0.00417$, the FRM behaves similarly to the previous case with a reduced value of the kinking load $(\lambda=1.35)$. The nonmonotonic behavior of the total energy in Figure 6.6 matches that observed in other numerical models of variational fracture, when local minimizers or saddle points of the discrete energy functional are determined 

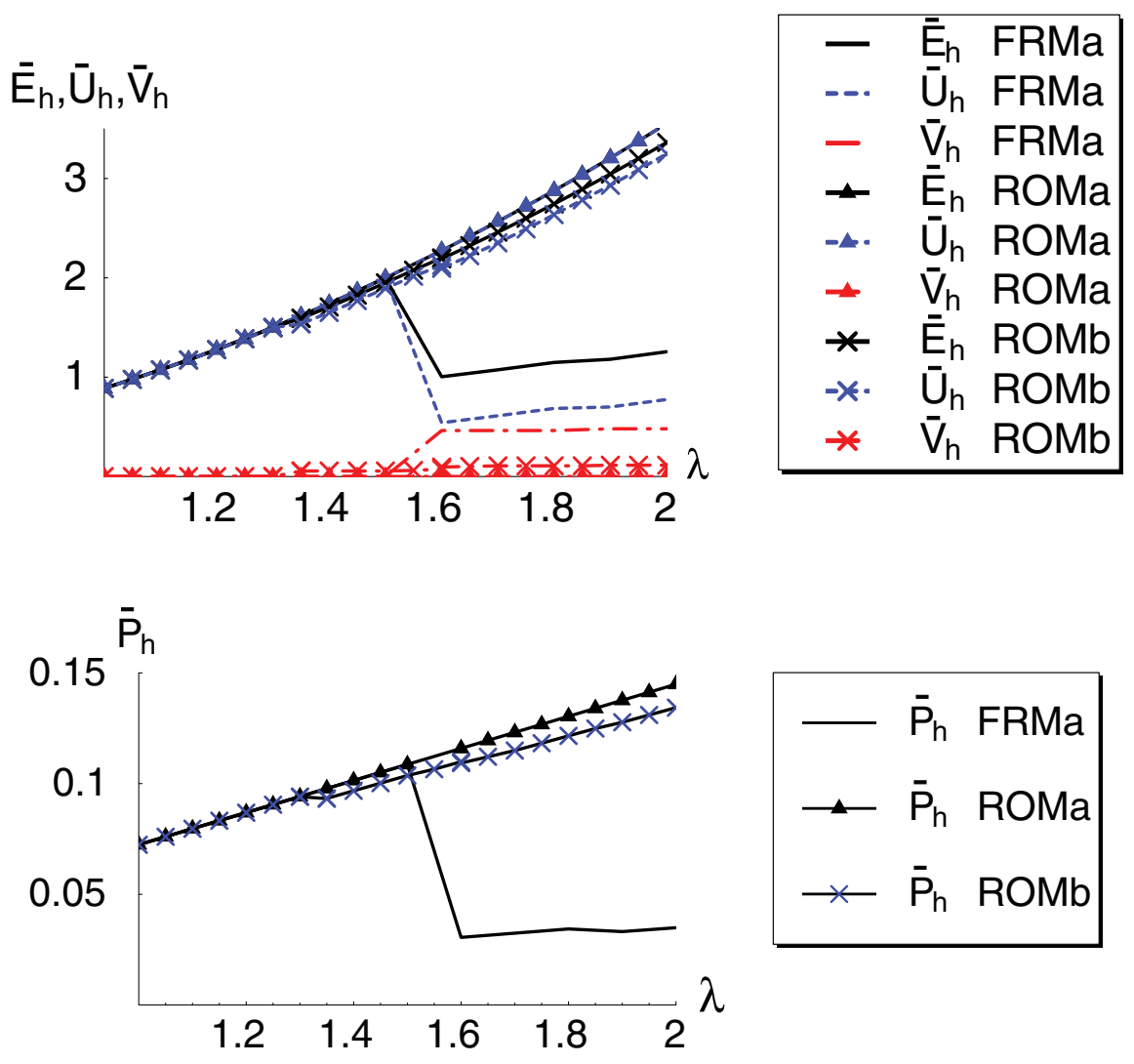

FIG. 6.6. Energy components and applied load vs. displacement multiplier $\lambda$ for $\alpha=45$ deg, $h=\varepsilon / 4=0.00625$ (a), and $h=\varepsilon / 4=0.00417$ (b).

(cf., e.g., [43], [8]). The ROM predicts kinking at $\lambda=2.8$ for $h=\varepsilon / 4=0.00625$ and at $\lambda=1.35$ for $h=\varepsilon / 4=0.00417$, a wavy crack profile, and kinking angle close to that estimated by Bourdin, Francfort, and Marigo in [10] $(\theta \approx-35$; cf. Figure 6.4). The same model also exhibits stable crack growth, and monotonic load vs. $\lambda$ response, despite the wavy nature of the crack trajectory (Figure 6.3). Clearly, the compliance of the cracked body is larger in the FRM than in the ROM. In particular, one observes that the ROM compliance during crack growth increases when passing from case (a) to case (b), i.e., when the mesh is refined. All the examined ROMs showed a snapwise crack growth, with alternation of crack extensions and purely elastic incremental deformations during the quasi-static loading process. The examined FRMs instead exhibited one or more steps of abrupt crack growth, up to the complete rupture of the plate. As already noticed, the differences between the two approaches are expected to progressively vanish as the mesh size goes to zero. However, to see this in the numerical simulations, it seems that very fine meshes have to be employed, which are beyond the scope of our current single processor based code. (Also compare the results of Bourdin [8], who uses a parallel code in order to be able to work with very 


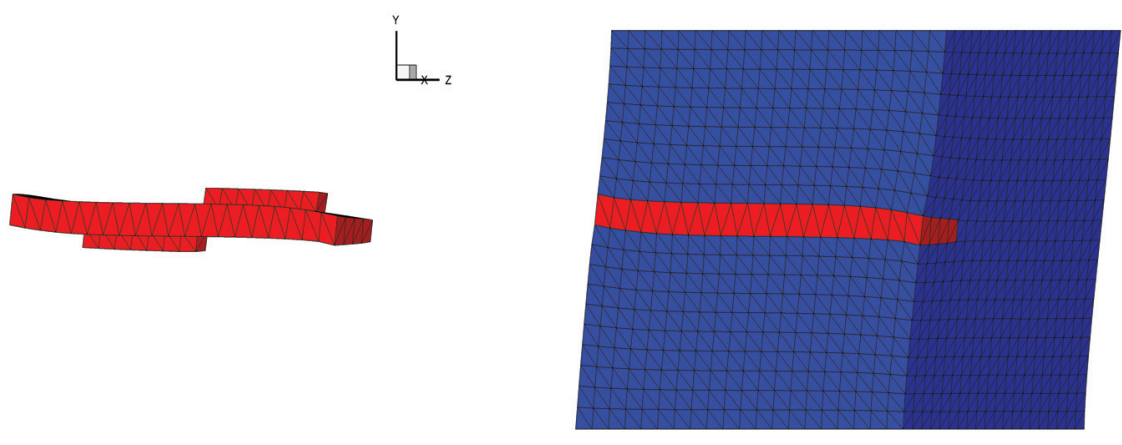

Step $1, \lambda=12.00$.

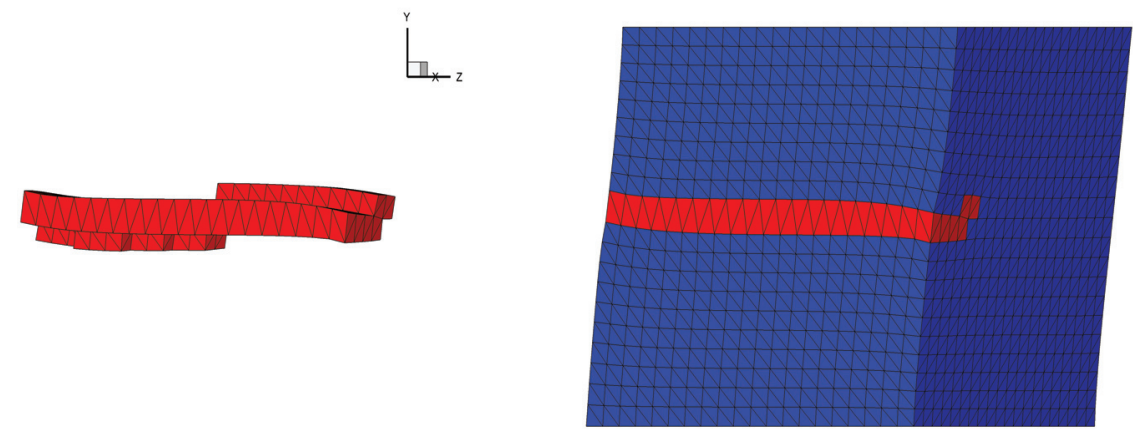

Step $3, \lambda=12.20$.

FIG. 6.7. Crack path and the deformed shape of a precracked plate loaded by combined mode I and mode III loading.

fine meshes.) We defer such an analysis to future work.

6.2. Crack propagation under combined mode I and mode III loading. Crack propagation under mixed mode I-III loading is a rather complex threedimensional problem that may exhibit singular features, such as wavy crack paths, riverbed patterns, and chaotic surface roughness. In the case of a planar crack, several experimental and theoretical studies have shown that the crack deviates from its initial plane when the ratio between mode III and mode I stress intensity factors exceeds a critical value, assuming a wavy profile with progressively increasing wavelength and amplitude (Sommer [52]; Gao [33]; Gao and Rice [34]; Xu, Bower, and Ortiz [53]). We show hereafter the crack pattern obtained for the plate of Figure 6.1 through the rank one eigendeformation model, for $\bar{u}_{3}=2.0 \times 10^{-4}, \bar{u}_{1}=1.0 \times 10^{-4}, \bar{u}_{2}=0$, $G=0.025 \times 10^{-6}, H_{3}=1$, and precrack length equal to $0.15 H_{1}$. A regular triangulation built on a $20 \times 20 \times 20$ uniform grid of nodes was employed (9282 nodes and 48000 elements), with $h=\varepsilon / 3=0.05, S=24, P=20, C=6$ (virtual crack extensions corresponding to cubes composed of six mesh simplices). In this case, the precrack was modeled introducing 504 prefractured elements with an S-shaped deviation from the $\left(x_{1}, x_{3}\right)$ plane. Figure 6.7 shows the progression of the crack growth for increas- 

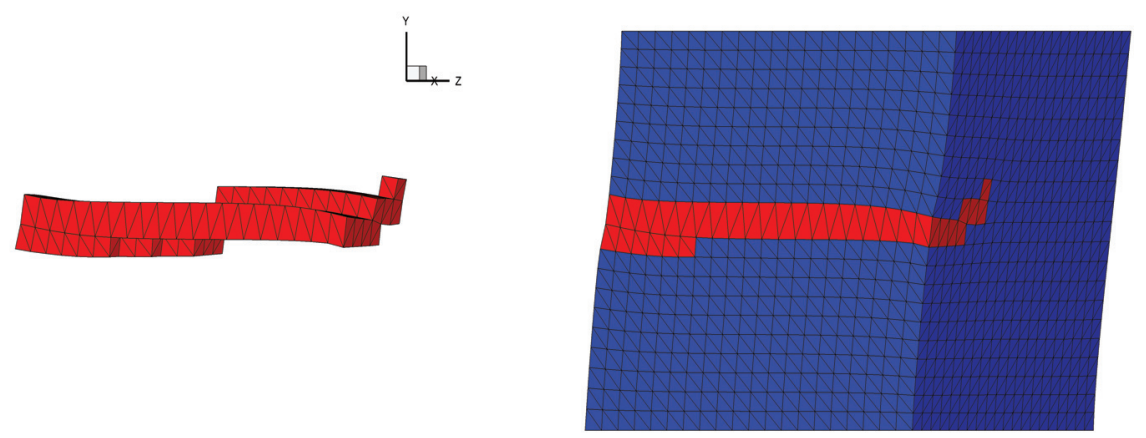

Step $5, \lambda=12.4$.

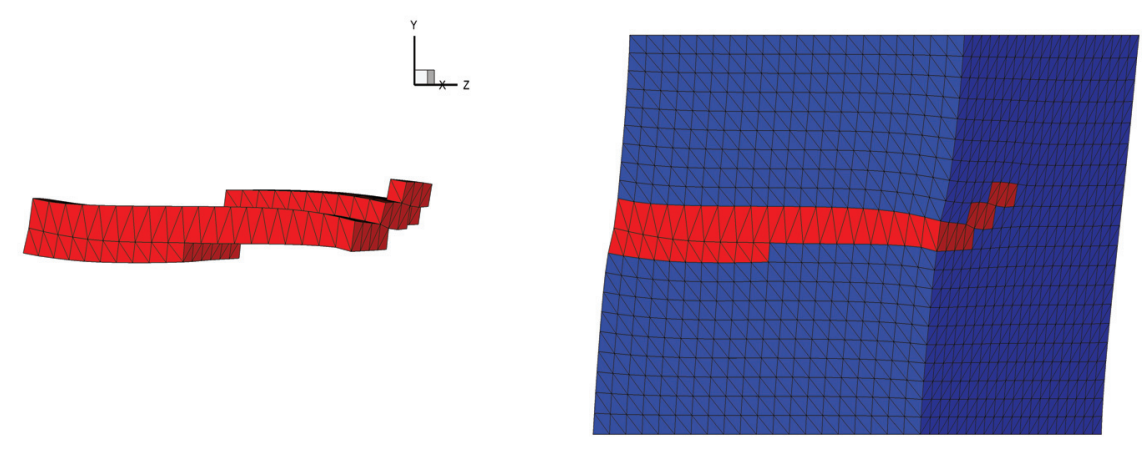

Step $7, \lambda=12.6$.

FIG. 6.7. (cont.).

ing values of the displacement multiplier $\lambda$ (displacement magnification factor $=25)$. One can notice that the adopted model is able to capture and suitably average the out-of-plane growth of the crack [53], with a relatively coarse mesh.

7. Summary and concluding remarks. We have developed an approximation scheme for the variational formulation of the theory of brittle fracture, which we term eigenfracture. The approximation scheme is built upon the use of eigendeformations to describe the cracks that occur in the body. The resulting approximating energy functionals depend on two fields: the displacement field and the eigendeformation field. The introduction of eigendeformations into the energy allows the displacement field to develop jumps at no cost in local elastic energy. However, this local relaxation requires the expenditure of a certain amount of fracture energy. We provide a construction, based on the consideration of $\varepsilon$-neighborhoods of the support of the eigendeformation field, for calculating the right amount of fracture energy associated with an eigendeformation field. The approximating functionals - and their finite element discretizations thereof-are then shown to converge variationally in the sense of $\Gamma$-convergence to the Griffith-type energy functional.

Whereas the description of displacement jumps across cracks by means of eigendeformations is relatively straightforward, a much more delicate issue concerns the 
precise amount of fracture energy to be accorded to an eigendeformation field. Specifically, an admissible fracture energy must ensure the $\Gamma$-convergence of the approximating functionals to the Griffith-type energy functional. As shown in the foregoing, the $\varepsilon$-neighborhood construction does supply an admissible fracture energy. The essential property of this construction is that, by "blowing up" the crack to its $\varepsilon$-neighborhood and scaling by $1 / 2 \varepsilon$ the Lebesgue measure of this set, it delivers an approximation of the $(d-1)$-dimensional Hausdorff measure of the crack set. In addition, the $\varepsilon$ neighborhood construction lends itself to an efficient numerical implementation, which reduces to aggregating the volumes of neighboring elements.

The method of eigenfracture, in particular, the $\varepsilon$-neighborhood construction, provides a simple and convenient device for overcoming deficiencies of conventional computational schemes for brittle fracture, including nonadaptive element erosion and cohesive elements. Thus, if the fracture energy is computed by naively adding element volumes scaled by the reciprocal of the element size, as commonly done in element-erosion methods [47], and if the interpolation spaces are constructed by regular refinement, the fracture energy may be overestimated in the limit. A similar overestimation of the fracture energy may occur when cracks are described by means of cohesive elements (cf., e.g., [14], [48]) and the mesh is refined uniformly. The $\varepsilon$ neighborhood construction eliminates the problem of overestimation of the fracture energy by effectively "smoothing out" the zig-zagging crack paths predicted by nonadaptive element erosion and cohesive elements. The proposed element erosion technique is driven by an energy descent criterion and thus differs from classical erosion methods based on local crack growth criteria (cf., e.g., [1]).

In closing, we point out a number of limitations of the present approach that suggest directions for future work. One obvious extension of the approach is to general cohesive models of fracture. While this might seem a simple extension, there are a number of technical difficulties that add challenge to the mathematical analysis. For instance, for general cohesive models the minimizers may exhibit a nonzero Cantor part of the deformation, though, interestingly, some models of damage rule this possibility out [25]. The presence of a Cantor part of the deformation may be physical in some cases and be related to processes of distributed damage [15]. Dealing effectively with these possibilities would require us to either show that the Cantor part of the deformation vanishes for certain cohesive models or to extend the $\varepsilon$-neighborhood construction to more general scalings. Another extension of the method concerns the consideration of the dissipative and irreversible aspects of fracture. In order to apply the methods of the calculus of variations to this case one requires a minimum principle that characterizes entire trajectories, or crack paths, of the system. Energydissipation functionals of the type recently proposed by Mielke and Ortiz [40] have this property. An analysis of fracture paths from front kinetics based on energydissipation functionals, including their rate-independent relaxation, has recently been carried out by Larsen, Ortiz, and Richardson [38]. However, the application of this or other minimum principles to the development of provably convergent numerical schemes for crack tracking remains at present an open question.

Acknowledgments. We thank M. Negri and C. Larsen for stimulating discussions on approximation schemes for fracture problems. We gratefully acknowledge the support of the Department of Energy through Caltech's ASC/ASAP Center for Simulating the Dynamic Response of Materials. F. F. gratefully acknowledges the support of the Italian Fulbright Commission and the Council for International Exchange of Scholars. 


\section{REFERENCES}

[1] A. Al-Ostaz And I. Jasiuk, Crack initiation and propagation in materials with randomly distributed holes, Engrg. Fracture Mech., 58 (1997), pp. 395-420.

[2] L. Ambrosio and V. M. Tortorelli, Approximation of functionals depending on jumps by elliptic functionals via $\Gamma$-convergence, Comm. Pure Appl. Math., 43 (1990), pp. 999-1036.

[3] L. Ambrosio and V. M. Tortorelli, On the approximation of free discontinuity problems, Boll. Un. Mat. Ital. B (7), 6 (1992), pp. 105-123.

[4] L. Ambrosio, A. Coscia, and G. Dal Maso, Fine properties of functions with bounded deformation, Arch. Rational Mech. Anal., 139 (1997), pp. 201-238.

[5] L. Ambrosio, N. Fusco, and D. Pallara, Functions of Bounded Variation and Free Discontinuity Problems, Oxford University Press, Oxford, UK, New York, 2000.

[6] M. Angelillo, E. Babilio, and A. Fortunato, A numerical method for fracture of rods, in Mechanical Modelling and Computational Issues in Civil Engineering, Lect. Notes Appl. Comput. Mech. 23, M. Frémond and F. Maceri, eds., Springer-Verlag, Berlin, 2005, pp. $277-292$.

[7] G. Bellettini, A. Coscia, and G. Dal Maso, Compactness and lower semicontinuity properties in $S B D(\Omega)$, Math. Z., 228 (1998), pp. 337-351.

[8] B. Bourdin, Numerical implementation of the variational formulation of brittle fracture, Interfaces Free Bound., 9 (2007), pp. 411-430.

[9] B. Bourdin and A. Chambolle, Implementation of an adaptive finite element approximation of the Mumford-Shah functional, Numer. Math., 85 (2000), pp. 608-646.

[10] B. Bourdin, G. A. Francfort, and J. J. Marigo, Numerical experiments in revisited brittle fracture, J. Mech. Phys. Solids, 48 (2000), pp. 797-826.

[11] A. Braides, Approximation of Free-Discontinuity Problems, Springer, Berlin, Heidelberg, New York, 1998.

[12] A. Braides, Г-Convergence for Beginners, Oxford University Press, Oxford, UK, 2002.

[13] A. Braides and G. Dal Maso, Nonlocal approximation of the Mumford-Shah functional, Calc. Var. Partial Differential Equations, 5 (1997), pp. 293-322.

[14] G. T. CAmacho And M. ORTiz, Computational modelling of impact damage in brittle materials, Internat. J. Solids Structures, 33 (1996), pp. 2899-2938.

[15] A. Carpinteri, B. Chiaia, and P. Cornetti, On the mechanics of quasi-brittle materials with a fractal microstructure, Engrg. Fracture Mech., 70 (2003), pp. 2321-2349.

[16] A. Chambolle, A density result in two-dimensional linearized elasticity, and applications, Arch. Ration. Mech. Anal., 167 (2003), pp. 211-233.

[17] A. Chambolle, An approximation result for special functions with bounded deformation, J. Math. Pures Appl. (9), 83 (2004), pp. 929-954.

[18] A. Chambolle, Addendum to: "An approximation result for special functions with bounded deformation": The N-dimensional case, J. Math. Pures Appl. (9), 84 (2005), pp. 137-145.

[19] A. Chambolle and G. Dal Maso, Discrete approximation of the Mumford-Shah functional in dimension two, M2AN Math. Model. Numer. Anal., 33 (1999), pp. 651-672.

[20] G. Cortesani and R. TOader, A density result in $S B V$ with respect to non-isotropic energies, Nonlinear Anal., 38 (1999), pp. 585-604.

[21] G. CoRTESAni And R. TOAder, Implementation of an adaptive finite element approximation of the Mumford-Shah functional, Numer. Funct. Anal. Optim., 18 (1997), pp. 921-940.

[22] B. Cotterell, On brittle fracture paths, Int. J. Fracture, 1 (1965), pp. 96-103.

[23] B. Cotterell and J. R. Rice, Slightly curved or kinked cracks, Int. J. Fracture, 16 (1980), pp. $155-169$.

[24] G. Dal Maso, An Introduction to $\Gamma$-Convergence, Birkhäuser, Boston, Basel, Berlin, 1993.

[25] G. Dal Maso and A. Garroni, Gradient bounds for minimizers of free discontinuity problems related to cohesive models in fracture mechanics, Calc. Var. Partial Differential Equations, 31 (2008), pp. 137-145.

[26] G. Dal Maso and R. Toader, A model for the quasi-static growth of brittle fractures: Existence and approximation results, Arch. Ration. Mech. Anal., 162 (2002), pp. 101-135.

[27] G. Dal Maso, G. A. Francfort, and R. Toader, Quasistatic crack growth in nonlinear elasticity, Arch. Ration. Mech. Anal., 176 (2005), pp. 165-225.

[28] E. De Giorgi and L. Ambrosio, Un nuovo funzionale del calcolo delle variazioni, Atti Accad. Naz. Lincei Rend. Cl. Sci. Fis. Mat. Natur. (8), 82 (1988), pp. 199-210.

[29] F. Erdogan and G. C. SiH, On the crack extension in plates under plane loading and transverse shear, J. Basic Engng., 85 (1963), pp. 519-527.

[30] G. Francfort And J.-J. Marigo, Revisiting brittle fracture as an energy minimization problem, J. Mech. Phys. Solids, 46 (1998), pp. 1319-1342.

Copyright (c) by SIAM. Unauthorized reproduction of this article is prohibited. 
[31] G. A. Francfort And C. J. LARSEn, Existence and convergence for quasi-static evolution in brittle fracture, Comm. Pure Appl. Math., 56 (2003), pp. 1465-1500.

[32] F. Fraternali, Free discontinuity finite element models in two-dimensions for in-plane crack problems, Theor. Appl. Fract. Mech., 47 (2007), pp. 274-282.

[33] H. GAO, Three-dimensional slightly nonplanar cracks, J. Appl. Mech., 59 (1992), pp. 335-343.

[34] H. GAO AND J. R. RICE, Shear stress intensity factors for a planar crack with slightly curved front, J. Appl. Mech., 53 (1986), pp. 774-778.

[35] A. Giacomini, Ambrosio-Tortorelli approximation of quasi-static evolution of brittle fractures, Calc. Var. Partial Differential Equations, 22 (2005), pp. 129-172.

[36] A. Giacomini And M. Ponsiglione, Discontinuous finite element approximation of quasistatic crack growth in finite elasticity, Math. Models Methods Appl. Sci., 16 (2006), pp. 77-118.

[37] C. J. LARSEN, E-stability and local minimality, to appear.

[38] C. J. Larsen, M. Ortiz, and C. L. Richardson, Fracture paths from front kinetics: Relaxation and rate-independence, Arch. Ration. Mech. Anal., submitted.

[39] L. Lussardi And M. Negri, Convergence of nonlocal finite element energies for fracture mechanics, Numer. Funct. Anal. Optim., 28 (2007), pp. 83-109.

[40] A. Mielke And M. Ortiz, A class of minimum principles for characterizing the trajectories and the relaxation of dissipative systems, ESAIM Control Optim. Calc. Var., 14 (2008), pp. $494-516$.

[41] J. R. Munkres, Elements of Algebraic Topology, Addison-Wesley, Menlo Park, CA, 1984.

[42] T. MurA, Mechanics of Defects in Solids, Martinus Nijhoff, Dordrecht, The Netherlands, 1987.

[43] M. NEGRI, Finite element approximation of the Griffith's model in fracture mechanics, Numer. Math., 95 (2003), pp. 653-687.

[44] M. NegRI, A discontinuous finite element approximation of free discontinuity problems, Adv. Math. Sci. Appl., 15 (2005), pp. 283-306.

[45] M. NEGRI, A non-local approximation of free discontinuity problems in $S B V$ and $S B D$, Calc. Var. Partial Differential Equations, 25 (2006), pp. 33-62.

[46] M. NEGRI, Convergence analysis for a smeared crack approach in brittle fracture, Interfaces Free Bound., 9 (2007), pp. 307-330.

[47] M. Ortiz and A. E. Giannakopoulos, Crack-propagation in monolithic ceramics under mixed-mode loading, Int. J. Fracture, 44 (1990), pp. 233-258.

[48] M. Ortiz And A. Pandolfi, A class of cohesive elements for the simulation of threedimensional crack propagation, Internat. J. Numer. Methods Engrg., 44 (1999), pp. $1267-$ 1282.

[49] C. C. Radon, P. S. Lever, And L. E. Culver, Fracture toughness of PMMA under biaxial stress, in Fracture, Vol. 3, UW Press, Waterloo, ON, Canada, 1977, pp. 1113-1118.

[50] G. C. Sin, Mechanics of Fracture Initiation and Propagation, Kluwer Academic Publishers, Dordrecht, The Netherlands, 1991.

[51] G. C. SiH, Strain energy density factor applied to mixed mode crack problems, Int. J. Fracture, 10 (1974), pp. 305-321.

[52] E. Sommer, Formation of fracture lances in glass, Engng. Fract., 1 (1969), pp. 539-536.

[53] G. XU, A. F. Bower, AND M. Ortiz, An analysis of non-planar crack growth under mixed mode loading, Internat. J. Solids Structures, 16 (1994), pp. 2167-2193.

Copyright $@$ by SIAM. Unauthorized reproduction of this article is prohibited. 